\title{
Tadpole Galaxies in the Hubble Ultra Deep Field
}

\author{
Bruce G. Elmegreen \\ IBM Research Division, T.J. Watson Research Center, 1101 Kitchawan Road, Yorktown \\ Heights, NY 10598 \\ bge@watson.ibm.com \\ Debra Meloy Elmegreen \\ Vassar College, Dept. of Physics \& Astronomy, Box 745, Poughkeepsie, NY 12604 \\ elmegreen@vassar.edu
}

\begin{abstract}
Tadpole galaxies have a head-tail shape with a large clump of star formation at the head and a diffuse tail or streak of stars off to one side. We measured the head and tail masses, ages, surface brightnesses, and sizes for 66 tadpoles in the Hubble Ultra Deep Field (UDF), and we looked at the distribution of neighbor densities and tadpole orientations with respect to neighbors. The heads have masses of $10^{7}-10^{8} M_{\odot}$ and photometric ages of $\sim 0.1$ Gyr for $z \sim 2$. The tails have slightly larger masses than the heads, and comparable or slightly older ages. The most obvious interpretation of tadpoles as young merger remnants is difficult to verify. They have no enhanced proximity to other resolved galaxies as a class, and the heads, typically $<0.2 \mathrm{kpc}$ in diameter, usually have no obvious doublecore structure. Another possibility is ram pressure interaction between a gas-rich galaxy and a diffuse cosmological flow. Ram pressure can trigger star formation on one side of a galaxy disk, giving the tadpole shape when viewed edge-on. Ram pressure can also strip away gas from a galaxy and put it into a tail, which then forms new stars and gravitationally drags along old stars with it. Such an effect might have been observed already in the Virgo cluster. Another possibility is that tadpoles are edge-on disks with large, off-center clumps. Analogous lop-sided star formation in UDF clump clusters are shown.
\end{abstract}

Subject headings: galaxies: evolution — galaxies: formation — galaxies: highredshift 


\section{Introduction}

Elongated galaxies with bright clumps at one end are visible in deep field images taken with HST or from the ground. van den Bergh et al. (1996) called them "tadpole" galaxies. Abraham et al. (1996) found that $13.4 \% \pm 1.6 \%$ of Hubble medium-deep-field galaxies showed tidal-like distortions or tails, compared to $8.7 \% \pm 1.0 \%$ locally. As part of a general survey of young galaxy morphology, we compiled a catalog (Elmegreen et al. 2005) of 97 tadpole galaxies in the ACS images of the Hubble Ultra Deep Field (UDF; Beckwith et al. 2006). We also determined photometric redshifts for these and other types larger than 10 pixels in diameter (Elmegreen et al. 2007). In the redshift range from 1 to 4, tadpoles represented $10 \%$ of the total count of such galaxies, comparable to the fraction of chain galaxies $(13.4 \%)$ and double galaxies (16.7\%), smaller than the clump-cluster fraction (33.4\%) and spiral galaxy fraction $(21.4 \%)$, and larger than the elliptical galaxy fraction (4.6\%). The spirals and ellipticals could be partially obscured by bandshifting, considering that these more conventional types tend to be intrinsically redder than the clumpy types.

Rhoads et al. (2005) studied a galaxy somewhat like a tadpole, UDF 5225, which they considered to have a core and a plume component, like a tadpole head and tail. We classified UDF 5225 as a chain galaxy, because the "plume" has three clumps in it. Rhoads et al. measured the spectrum throughout and found continuum and $\mathrm{L} \alpha$ emission at redshift $z=5.4$. They concluded that the galaxy was a merger with star formation in the core and in knots along a tidal tail.

Straughn et al. (2006) and Windhorst et al. (2006) identified 165 tadpole galaxies in the UDF using an automated search algorithm. They showed that the photometric redshift distribution for tadpoles was about the same as that of field galaxies, with tadpoles representing $6 \%$ of the total. Following simulation results by Springel et al. (2005a, b) , who found a tadpole phase 0.7-1.5 Gyr after a merger and the epoch of peak black hole accretion $\sim 1$ Gyr after that, Windhorst et al. compared the tadpole population to UDF galaxies with some AGN-type variability. They saw no overlap between the two populations, and suggested that this was consistent with the Springel et al. prediction about the relative timing of different phases. Straughn et al. also noted that every galaxy would have to undergo 10 to 30 such mergers since redshift $\sim 7$ to account for the tadpole fraction, given the relatively short time spent in this phase. They suggested that since tadpole shapes require a certain type of merger, the actual number of strong interactions or mergers per galaxy would have been larger.

Rawat et al. (2007) studied 39 Luminous Compact Galaxies that included 3 tadpoles with stellar $\log M / M_{\odot}=10.23,9.71$, and 10.67. They noted that the tadpole heads were bluer than the tails, and suggested that this whole class of luminous galaxies is evolving 
toward intermediate mass spirals, with major mergers playing an important role in the observed morphology. Mergers were inferred primarily from the presence of multiple cores with similar luminosities in the rest-frame B-band, or the presence of double red nuclei. One of their tadpoles has a double-core head.

De Mello et al. (2006a) show a tadpole galaxy in their Figure 16 and note that many compact galaxies in their UV-selected sample have tadpole morphologies. De Mello et al. (2006b) studied 268 UV-bright sources in parallel WFPC2 fields of the Great Observatories Origins Deep Survey (GOODS; Giavalisco et al. 2004) and visually classifying 93 with starburst SEDs. They found that $36 \%$ of the starbursts are tadpoles and $50 \%$ of the starbursts have another galaxy within a $5^{\prime \prime}$ area (which corresponds to a $20 \mathrm{kpc}$ radius at their average $z=1.5$ ). The half-light radii of the tadpoles was $1.6 \pm 0.4 \mathrm{kpc}$. Several are shown in their Figures 11, and 12 .

Here we use the ACS and NICMOS UDF fields to measure the head and tail masses, ages, surface densities, and sizes for 66 tadpoles in our morphology catalog. We find that tadpole heads are usually single at the resolution of the ACS. They look like clumps in clump-cluster galaxies, i.e., they are low-mass and young compared to whole $\mathrm{L}^{*}$ galaxies. Note that tadpoles in our survey are defined only by the presence of a bright clump of light at one end and a smooth, somewhat linear, tail extending away from the clump; they are not defined to have single-component heads. We discuss examples of tadpole heads with substructure in Section 4.2, these could be mergers or they could be complex structures from star formation in the heads.

We also look for an excess of neighbors around tadpoles. We compare the projected densities of galaxies around them with the projected densities of galaxies around random field galaxies in the UDF. No difference is evident, nor is there a consistent orientation of tadpoles relative to near-neighbor directions. A few tadpoles have double heads (examples are shown), but generally there is little off-axis structure, duplicity, or other evidence for mergers or interactions. This lack of evidence is consistent with the recent suggestion that galaxy mergers are less important for building galaxies than formerly thought. Major mergers thicken stellar disks in unacceptable ways (Bournaud \& Elmegreen 2009; Bournaud et al. 2009), and they are not essential for building galaxies in the presence of cold flows (Ocvirk et al. 2008; Dekel et al. 2009; Agertz et al. 2009; Kerês et al. 2009; Brooks et al. 2009).

Other possible origins for tadpole structure are gas stripping or star-formation triggering by ram pressure. The tail could be star-forming gas that was stripped from the head and accompanied by entrained older stars, or the head could be the leading surface of a low-mass disk that was compressed into star formation. Ram-pressure stripping and triggering are expected during galaxy motions through dense cosmological gas flows in the neighborhood. 
A related possibility is suggested by normal velocity gradients across the tails of several tadpoles observed by Förster Schreiber et al. (2009). These gradients suggest that the tadpoles are rotating disks like some clump-clusters, but with a single large clump instead of a half-dozen clumps. We suggest that the single clump could be the brightest part of a ring or a bright clump near the edge of a stellar disk, and we show examples of such galaxies.

We conclude with the suggestion that tadpoles could be a composite population with several physical origins. Further studies of gas and molecular abundances, internal dynamics, and galactic environment will be necessary to understand the tadpole shape more fully.

In what follows, Section 1 discusses the data, Section 2 contains the method of analysis for mass and age, Section 3.1 has the results for mass, age, and surface density, 3.2 looks at companions, 3.3 considers internal dynamics, and 3.4 has two examples of clustered tadpoles suggestive of environmental effects, as well as examples of what may be face-on tadpoles in the lop-sided disk interpretation, A discussion of possible models for tadpoles is in Section 4 , and the conclusions are in Section 5.

\section{Data}

The tadpole galaxies catalogued in Elmegreen et al. (2005) were studied for the present paper. There were 97 tadpoles in that catalog, all larger than 10 pixels in diameter. Here we excluded from our photometric study the cases that looked obviously like mergers, were contaminated by other galaxies, or seemed to have spiral structure, which puts them in a different morphology category. Obvious mergers are those with disconnected parts, multiple bright regions with clear separations, multiple tails and other classical tidal features, and off-axis emission. Objects with such features are considered to be mergers in the classical sense. These features make them look different from the simplest tadpoles, which have single bright heads and single tails, all on approximately the same axis. We discuss in section 4.2 tadpoles with structured heads that might be late-stage mergers, although even these have single tails and little off-axis structure. We also discuss in section 4.2 tadpoles with slightly curved tails. These could be interacting tadpole galaxies with unknown companions that accelerate the main tadpoles in the off-axis direction. They also do not look like conventional mergers, so they are included in our main study here. We discuss them specifically later as special cases in order to consider the possibility that some tadpoles might be interacting or mergers even though they do not look like it in the conventional sense.

We used photometric redshifts from Rafelski et al. (2009), which includes ground-based UV measurements, and excluded tadpoles that were not in the Rafelski et al. compilation. 
Other tadpoles were excluded for faintness, particularly in B-band for high- $z$ dropouts. For the remaining sample of 66 tadpoles, the $\mathrm{AB}$ magnitudes and number of pixels were determined for the heads and for the prominent regions of the tails using the four ACS passbands, $B_{435}, V_{606}, i_{775}$, and $z_{850}$. Boxes around the objects were defined using the IRAF task imstat. Typically the object boundaries were at a level about $10 \sigma$ above the noise. Sky was not subtracted because it is essentially zero.

NICMOS observations of the UDF (Thompson et al. 2005) also included tadpole galaxies. For 37 cases with Rafelski et al. redshifts, we measured the J and H-band magnitudes of the heads, along with the $B_{435}, V_{606}, i_{775}$, and $z_{850}$ ACS magnitudes of the same heads on images convolved to the same resolution as NICMOS.

Zeropoint conversions for each filter were taken from the online handbooks. Magnitude measurement errors are estimated to be about 0.1 mag. Boxes rather than circles were used to define magnitudes because the regions were sometimes elongated. The same box position was used for each passband. Head and tail colors typically varied by less than 0.05 mag for different box placements or sizes.

Figure 1 shows a collection of 8 tadpole galaxies at ACS resolution in color, from the UDF Sky Walker 1 (on the left), in ACS $i_{775}$ (second from the left) and NICMOS $H$ (second from the right, with pixels three times larger than in Sky Walker). On the right is an intensity scan through the length of the galaxy as viewed by the ACS in $i_{775}$ band. This class of objects has a bright head and a diffuse tail. Many tadpoles have slightly different morphologies; some have diffuse emission that is not particularly elongated to the side of a clump. Sometimes the tail has a clump, or one could interpret the structure as a double head. In all cases, there is a bright clump at the end of a diffuse region. Because photometric redshifts were determined for whole tadpole galaxies and not the heads and tails separately, we cannot tell if the different parts of a tadpole are really separate galaxies. As a result, we interpret possible differences in component redshift as differences in color, age, or extinction.

Figure 2 shows the redshift distribution of apparent $\mathrm{AB}$ magnitude in $i_{775}$ band at full ACS resolution for the tadpole heads and tails, in $i_{775}$ for the heads at the NICMOS resolution, and in H-band for the heads from NICMOS. We reach a limiting $i_{775}$ magnitude of $\sim 29.5$. Absolute magnitudes are shown by the dotted lines, using distance moduli from a $\Lambda$ CDM cosmology (Spergel et al. 2003). The NICMOS low-resolution measurements are systematically brighter than the ACS high-resolution measurements because of the inclusion of slightly more projected area at the lower resolution. The tails are systematically brighter than the heads at the same ACS resolution. Tails are often not observed well in NICMOS

\footnotetext{
${ }^{1}$ designed by K. Jahnke and S.F. Sánchez, AIP 2004
} 
so we do not discuss that measurement in this paper.

\section{Analysis}

Bruzual \& Charlot (2003) models of stellar population spectra were used to determine redshifted model colors for comparison with the observed colors of the heads and tails. A metallicity of 0.008 (equal to 0.4 solar) and the Chabrier IMF was assumed. We considered an exponentially decaying star formation history with separate start times and decay times for each clump and head. The decay times considered were, in Gyr, 0.01, 0.03, 0.1, 0.3, 1, 3, and 10. Intervening cosmological hydrogen absorption (Madau 1995) is included, as well as internal dust absorption using the wavelength dependence in Calzetti et al. (2000) with the short-wavelength modification in Leitherer et al. (2002). A $\Lambda$ CDM cosmology was assumed (Spergel et al. 2003). As mentioned above, photometric redshifts come from Rafelski et al. (2009).

The rms differences between the model colors and the observed colors were determined for each of a wide range of start times, decay times, and extinctions. These rms differences were binned into groups with values incremented by 0.1 . Among the groups with the lowest rms differences, weighted average values of the start times (ages), decay times, and extinctions were determined. The weighting parameter is $\exp \left(-0.5 \chi^{2}\right)$ where $\chi^{2}$ is the sum of the squares of the color differences divided by the rms errors in the colors, as determined from the pixel counts used for the magnitudes. The mass follows from the observed $i_{775}$ magnitude in comparison to the model. The surface density of a tail is taken to be the tail mass divided by the projected area of the measured region. For more details of this method, see Elmegreen et al. (2009a, b).

\section{Results}

\subsection{Masses and Ages}

The masses of the tadpole heads and tails are shown as functions of $z$ in the left panel of Figure 3. For comparison, clump masses in UDF clump-cluster and chain galaxies are shown in the middle panel, and bulge masses in the clump clusters and chains that have

bulges are shown on the right (from Elmegreen et al. 2009a). Bulges are defined to be the reddest, and often the most luminous clumps; they are usually, but not always, centralized. The tadpole head and clump-cluster bulge masses were evaluated twice, once with J and H NICMOS observations and 4-band ACS observations blurred to NICMOS resolution (red 
circles), and again with only the 4-band ACS observations at full resolution (blue dots). The small black dots in the middle and right-hand panels are clumps and bulges, respectively, in GOODS galaxies (Elmegreen et al. 2009b), which have 4 ACS bands and extend to $z \sim 1$. There are relatively few GOODS measurements compared to UDF measurements because clump clusters are extremely rare at the low redshifts of the GOODS survey. The 4-band ACS measurements of tadpole head mass with $0.03^{\prime \prime}$ pixel size (blue dots in the left panel) are slightly smaller than the J-H NICMOS + ACS measurements with $3 \times$ larger pixels (red circles). This is partly because the larger pixels include more peripheral light, and partly because the NICMOS IR bands include more old stars.

The average trends for $\log M$ (in $M_{\odot}$ ) versus $z$ on the left in Figure 3 are somewhat linear. We fit them to the function $\log M=A+B z$ with $A=5.86 \pm 0.50$ and $6.78 \pm 0.73$ for ACS-only determinations of the head mass and ACS+NICMOS determinations of the head mass, respectively. The slopes are $B=0.51 \pm 0.16$ and $0.39 \pm 0.21$, respectively. Error bars for this linear fit are $95 \%$ confidence intervals. The general decrease in mass at low $z$ is a selection effect related to size and cosmological surface brightness dimming (Elmegreen et al. 2009b). For $z>1$, the average $\log$ of the tadpole head mass calculated with ACS only is $7.69 \pm 0.69$. The average log mass measured with ACS of 906 UDF clump cluster and chain clumps at $z>1$ in the middle panel is $7.22 \pm 1.34$. The average $\log$ mass for 23 UDF clump cluster and chain bulges at $z>1$ in the right-hand panel, calculated with ACS only, is $8.11 \pm 0.43$. Thus the tadpole heads at $z>1$ have an average mass that is larger than the average clump cluster clump mass at the same redshift by a factor of $\sim 3$, and smaller than the average clump cluster bulge mass by a factor of $\sim 2.6$. The tadpole heads are not as massive as whole clump clusters or chain galaxies in our surveys, which typically contain $\sim 10^{10} M_{\odot}$ or more in stars.

Tail masses in Figure 3 were determined from rectangular areas enclosing the whole tail, including some apparent sky regions but not the head, to the extent that excluding the head was possible. A linear fit to the $\log$ of the tail mass in Figure 3 gives $\log M=$ $(6.93 \pm 0.64)+(0.36 \pm 0.20) z$. The sum of the head and tail for individual galaxies scales with redshift as $\log M_{\text {sum }}=(6.82 \pm 0.54)+(0.48 \pm 0.17) z$. At $z=2$, this summed mass is $6 \times 10^{7} M_{\odot}$. The average of the log of the ratio of the tail to the head mass for individual galaxies is $0.67 \pm 0.86$, meaning that tails are more massive than heads by a factor of $\sim 4.7$.

Root mean square deviations in the logarithm of the mass are shown in Figure 4 on the left, averaged over bins of redshift for all of the objects. The three panels are for different types of measurements: ACS measurements of the heads (top), ACS+NICMOS measurements of the heads (middle), and ACS measurements of the tail (bottom). The rms values of $\log M$ represent deviations among the mass results for all of the models within the 
lowest bins of rms deviations in the color, as discussed above. The rms values are lowest for the ACS+NICMOS measurements, and there is a slight increase in rms with redshift.

Figure 5 shows the tadpole head ages in comparison to the ages of clumps and bulges in clump cluster and chain galaxies. The green dashed lines represent the age of the universe as a function of redshift. Derived ages are less certain than masses because of an ambiguity between reddening from age and reddening from extinction (these two effects compensate for each other in the case of mass). Root mean square values for the logarithm of the age are shown on the right in Figure 4; they are larger than the $\log M \mathrm{rms}$ values by about $50 \%$. The ages of the tadpole heads span the same large range as the ages of the bulges in clump clusters and chain galaxies. The heads are older than the bulk of the clumps at low redshift, but comparable to the ages of clumps at $z \sim 2-4$, which are also comparable to the ages of bulges there.

Figure 6 shows the projected mass surface densities of tadpole tails versus redshift (black dots), compared with the mass surface densities of the interclump media in four types of galaxies in the GOODS fields, from Elmegreen et al. (2009b). These four types are labeled in the panels. Two-arm spirals and flocculent spirals resemble spiral galaxies today; clump clusters are composed primarily of several massive clumps of star formation with little interclump medium, and clump clusters with red disks have the same clumpy star formation but there is a red old-star component between them. We suggested in Elmegreen et al. $(2009 \mathrm{~b})$ that there is an evolutionary sequence from clump clusters with no evident interclump medium to clump clusters with red interclump media, to spirals, on the basis of the mass surface density and age of the interclump regions, in addition to the presence of bulges in spiral galaxies, which seems to be a later phase than a clump cluster (Elmegreen et al. 2009a). The GOODS galaxies extend to $z \sim 1$ while the UDF galaxies extend further in redshift. Both have an increasing trend of surface density with redshift from selection effects related to cosmological surface brightness dimming. The rms errors in the fits to the surface density are the same as the rms errors in the fits to the mass.

The mass surface densities in tadpole tails were determined from rectangular regions entirely enclosed in the tails, so they represent the values in the tail centers. Figure 6 indicates that the mass surface densities in tadpole tails are generally less than the mass surface densities in spiral and flocculent galaxies, by a factor of $\sim 10$. Thus the tails are not normal galaxy disks (but they could be low surface-brightness disks). The tadpole tails are also slightly lower in surface density than the red parts of clump clusters with red interclump regions, and also lower than the interclump regions between the clumps of pure clump clusters. These trends are evident only in the small region of overlap in the figure, which is at low redshift. We do not have similar measurements for interclump regions in 
high redshift clump clusters from the UDF because these regions are generally very faint. We see the tadpole tails in the UDF because they are isolated from bright clumps.

Figure 7 shows the ages of the tadpole tails compared with the ages of the interclump regions of the 4 types of galaxies in our GOODS study. These region are compared because they are all somewhat diffuse and outside the obvious star formation clumps. The figure indicates that all of the ages are about the same, in the range from 0.01 to 1 Gyr, with a concentration around 0.1 Gyr for the high-redshift tadpole tails.

A histogram of the difference in the log of the age between the head and the tail for individual galaxies (log head age - log tail age) is shown in Figure 8. The average difference is $-0.3 \pm 0.9$, suggesting slightly younger heads than tails, but this difference is essentially zero within the errors.

The head and tail densities can be estimated from the masses, column densities, and sizes. The average size of a tadpole head is measured to be $\sim 0.3 \pm 0.4 \mathrm{kpc}$, corrected for the ACS point spread function (Section 4.2). For a typical mass of $10^{8} M_{\odot}$ (Figure 3 ), the head has a density of luminous stars $\sim 3.7 M_{\odot} \mathrm{pc}^{-3}$, and a column density $\sim 1100 \mathrm{M}_{\odot} \mathrm{pc}^{-2}$. We can do this more accurately considering each galaxy separately and counting only those with resolved heads. Then the average head density is $2.0 \pm 4.4 M_{\odot} \mathrm{pc}^{-3}$, and the average head column density is $880 \pm 1800 M_{\odot} \mathrm{pc}^{-2}$. The transverse size of a tail is about the same as the head size. The average tail surface density is $69 \pm 83 M_{\odot} \mathrm{pc}^{-2}$ in Figure 6, so the average tail density is this surface density divided by the typical size, or $0.2 M_{\odot} \mathrm{pc}^{-3}$. If the tail density is determined for each galaxy separately, then the average is $0.12 \pm 0.13 M_{\odot}$ $\mathrm{pc}^{-3}$, a factor of 16 less than the head density.

Histograms of the extinctions in the heads and tails are shown in Figure 9. These were obtained from the stellar population fits to the 4 ACS passbands, along with the masses, ages, and star formation decay times (not shown). The average extinctions for the heads and tails are $1.7 \pm 1.3 \mathrm{mag}$. and $1.7 \pm 1.2 \mathrm{mag}$., respectively. Extinctions and ages are the most uncertain parts of the model fits. The ACS+NICMOS fits to the head regions also gave extinctions, but because NICMOS could not detect most of the tails and was not used for the tail model fits, this figure shows only the ACS extinctions in comparing the heads and the tails.

\subsection{Companions}

We studied the environments of tadpoles by counting companions within a fixed restframe projected separation and a fixed redshift interval. The companions were UDF galaxies 
larger than 100 square pixels in area, since the tadpoles themselves are larger than 10 pixels in length. We considered only the UDF galaxies with photometric redshifts in the latest compilation based on supplemental uv data (Rafelski et al. 2009). This is consistent with our use of the same redshift catalog for the tadpoles themselves.

Figure 10 (left) shows, in red, a histogram of the number of companions to tadpole galaxies within a projected distance of $100 \mathrm{kpc}$ at the distance of the tadpole and within a redshift interval of 0.2 . The right hand axis is used. It also shows, in blue, a histogram of the number of companions for all galaxies in the same UDF catalog used for the companions, i.e., larger than $100 \mathrm{px}^{2}$ area with redshifts in Rafelski et al. (2009). The left-hand axis is used for this. The histograms are very similar, suggesting that the number distribution of companions around tadpole galaxies is about the same as the number distribution of companions around any other large galaxy. This would imply that tadpoles do not have an excess or lack of companions compared to other galaxies. The right-hand panel shows the same neighbor counts in a cumulative distribution, from which a Kolmogorov-Smirnov test was performed. We find a $74 \%$ probability that the tadpole companions and the field-galaxy companions are drawn from the same near-neighbor distributions. Other separations and redshift intervals give the same result: for separations within $100 \mathrm{kpc}$ and a redshift interval of 0.8 , the KS probability is $58 \%$; for $200 \mathrm{kpc}$ and $\Delta z=0.2,53 \%$, and for $200 \mathrm{kpc}$ and $\Delta z=0.8,64 \%$. Also for these other limits, the peaks in the near-neighbor histograms shift toward more neighbors, as expected for the bigger space volumes considered. For the 4 cases, respectively, the peaks of the distributions are centered at 1, 6, 7, and 26 companions. In a more extreme case with a separation of $50 \mathrm{kpc}$ and a redshift interval of 0.2 , there are very few galaxies in the neighborhood: the peak in the distribution of the number of companions is at 0 companions and the KS probability that the tadpoles are drawn from the same distribution as the field galaxies is nearly $100 \%$.

To further test the similarity of tadpole neighborhoods with those of field galaxies, we added fake galaxies around the tadpoles with certain probabilities to see how this affects the KS tests. Recall that for $100 \mathrm{kpc}$ distance and a redshift interval of 0.2 , the KS probability that the real tadpole neighbors and the field neighbors are from the same distribution is $74.3 \%$. If we add one extra companion to $2 \%$ of the tadpoles, then this probability drops to $26.6 \%$. For an extra companion added to $5 \%$ of the tadpoles, it drops to $9.3 \%$, for $10 \%$ of the tadpoles, $0.084 \%$, and for $50 \%$ of the tadpoles, $0.0093 \%$. Thus we cannot tolerate an additional companion around even $5 \%$ of the tadpoles before the companion distribution begins to look significantly different from the field galaxy companion distribution. The reason for this is that additional companions shift the tadpole histograms in Figure [10 slightly to the right relative to the distribution for all galaxies. The histograms are so sharply peaked, however, than even a slight shift for the tadpoles causes the histogram to significantly exceed 
the field galaxy histogram at high numbers.

We conclude from these tests that tadpole galaxies have normal neighbor distributions; they are not significantly close to companions larger than 10 pixels in size. They could still be mergers, however, with the merged galaxies unresolved in the head for the majority of cases where only one head clump is observed.

The case of mergers is investigated next. Figure 11 shows the size distributions of the tadpole heads and tails. The head size is defined to be the square root of the difference between the area in pixels and the area of the FWHM of the point spread profile. The FWHM point spread profile was measured from stars at $V_{606}$ to be 3.08 pixels. The corrected angular size is converted into kpc using the redshift. The average head size (including those with effectively zero size because they are at the resolution limit) is $0.31 \pm 0.36 \mathrm{kpc}$. This is relatively small for a merger; each tadpole would have to have its merging sub-galaxy close to perigalacticon. The average tail length is $3.9 \pm 1.7 \mathrm{kpc}$, also small for a tidal tail by local standards.

We also looked for double-core or clumpy heads. From the list of tadpoles in Elmegreen et al. (2005), 13\% have something that might be called a double-core head, 3.3\% have multi-core (lumpy) heads, and 6.5\% have disky (elongated) heads. Figure 12 shows examples of tadpoles with clumpy heads or multiple-clump structure throughout. These multi-core cases could be interactions or mergers, or they could just be clumpy, single-galaxy heads. UDF 9543 has a wiggly tail, which is rare. The tadpole UDF 8614 in Figure 1 has a double-core head and wiggly tail too (when viewed with the right contrast, the two cores in the head are aligned with the tail). The wiggly tails in these two cases could be the result of variable external pressure forces or orbital motions inside the heads, which gravitationally drag the tails around with them. Most tadpoles in our present survey do not have double cores in their heads. Perhaps higher resolution observations will show more double cores.

Another consideration is the orientation of the tadpole tails relative to nearby galaxies. Figure 13 shows the distribution of angles, measured at the midpoint of the tail, between the midpoint of the head and the midpoint of a companion galaxy, versus the distance between the head and the companion normalized to $100 \mathrm{kpc}$ at the distance of the tadpole. Companions are considered within a redshift interval $\Delta z= \pm 0.2$. When this angle is $0^{\circ}$, the tadpole head points toward the companion, and when it is $180^{\circ}$, the head points away from the companion. These are projected angles, so the numbers of tadpole-companion combinations in each $45^{\circ}$ quadrant of this plot would be about equal for a random distribution of orientations. This appears to be the case, suggesting that tadpoles are not pointing in any particular direction relative to their companions, regardless of the companion distance. We found the same random orientations for various combinations of maximum projected 
separation and maximum redshift interval (i.e., separations of 100 and $200 \mathrm{kpc}$ and maximum $\Delta z$ of 0.2 and 0.8$)$.

Tadpole tails can be curved, flared, or clumpy. In 92 tadpoles from our UDF sample, $22 \%$ are flared and about half of these are also clumpy in the flared regions, $13 \%$ of the tails are curved and most of these have no significant clumps, and $21 \%$ are straight and clumpy. The rest $(42 \%)$ are straight without significant clumps. Curved tails have only minor curvature, around $30^{\circ}$ at most.

Companions to high redshift galaxies were also studied by Conselice \& Arnold (2009), who looked at ACS UDF dropout paired galaxies in $\mathrm{B}_{450}, \mathrm{~V}_{660}$, and $\mathrm{i}_{775}$ bands with magnitudes $z_{850}<28.5$. These samples correspond to redshifts equal to approximately 4 (320 galaxies), 5 (137 galaxies), and 6 (126 galaxies). They defined close pairs as two galaxies in the same dropout category with projected separations less than $20 \mathrm{kpc}$ (for $H_{0}=75 \mathrm{~km} \mathrm{~s}^{-1}$ $\left.\mathrm{kpc}^{-1}\right)$. The dropout pair fractions for the three passbands are $0.21 \pm 0.03,0.19 \pm 0.04$ and $0.16 \pm 0.05$, respectively. They also studied the asymmetry index for a subsample of these galaxies with $z_{850}<27.5(69,43$, and 21 galaxies, respectively) and found similar fractions with an asymmetry indicative of a merger: $0.23 \pm 0.05,0.19 \pm 0.05$, and $0.19 \pm 0.13$, respectively. In the present study, we have excluded galaxy pairs in our selection of tadpole shapes ("doubles" were a different morphology class in our UDF catalog). We also exclude galaxies with peculiarities or asymmetries that are not like tadpole shapes; tadpoles are well defined and usually symmetric around one axis. Thus the comparison with the work of Conselice \& Arnold is not straightforward. Overall, the tadpole fraction in the UDF (10\% in our catalog) is about half of the pair or asymmetry fractions found by Conselice \& Arnold. Our search for companions within $50 \mathrm{kpc}, 100 \mathrm{kpc}$, or $200 \mathrm{kpc}$ and a small range in redshifts covers a much larger volume than the paired companions searched by Conselice \& Arnold, which are within $20 \mathrm{kpc}$. Tadpoles are not, by definition, members of pairs or such strong mergers that they become highly distorted. Because there is also no evidence for an excess or lack of companions at $50 \mathrm{kpc}$ or beyond, the tadpoles do not seem to get their main structure from interactions.

Although we see no evidence for interactions, mergers, or nearby companions in most tadpole galaxies, we cannot rule out interactions and mergers as a cause for their structure. Interactions could involve objects smaller than 100 pixels in area or with uncertain redshifts, which were excluded from our neighbor list. They could all be late-stage mergers with unresolved double cores, although the tadpole fraction of $10 \%$ would require a high and continuous merger rate, as mentioned in the introduction. 


\subsection{Internal Dynamics}

Understanding the nature of tadpole galaxies requires dynamical information. Förster Schreiber et al. (2009) include 4 tadpoles in their SINFONI spectroscopic survey of high-redshift galaxies. These galaxies are SSA22a-MD41 and Q2343-BX389, shown in their Figure 24, and Q2346BX405, and Q2346-BX482, shown in their Figure 25. In order, their spectroscopic redshifts are $2.17,2.17,2.03$, and 2.26 , which are in the same range as our tadpoles. Their total stellar masses from SED fits are $0.72 \times 10^{10} M_{\odot}, 4.40 \times 10^{10} M_{\odot}, 1.58 \times 10^{10} M_{\odot}$, and $1.69 \times 10^{10} M_{\odot}$, which are larger than the masses of our tadpole heads by nearly 2 orders of magnitude (see Figure 31). Their dynamical masses from gas emission lines are, respectively, $6.9 \times 10^{10} M_{\odot}, 14 \times 10^{10} M_{\odot}, 2.8 \times 10^{10} M_{\odot}$, and $13 \times 10^{10} M_{\odot}$, which are larger than their SED stellar masses by factors of 9.6, 3.2, 1.8, and 7.7. These are reasonable factors for dark matter halos.

The higher masses in the Förster Schreiber et al. study reflect the brighter magnitude limits of their surveys. UDF tadpoles are small by comparison. The NICMOS $\mathrm{H}_{\mathrm{AB}}$ magnitudes of the heads of our tadpole galaxies range from 25 to 29 (see Figure 21). The $\mathrm{H}_{\text {Vega }}$ magnitudes of the first 2 tadpole galaxies from the Förster Schreiber et al. list above are $21.27 \pm 0.05 \mathrm{mag}$ and $21.75 \pm 0.10 \mathrm{mag}$, and for the last galaxy, $20.98 \pm 0.07 \mathrm{mag}$. Adding the magnitude correction of 1.31 (Stanway et al. 2005) to convert from Vega to AB magnitudes, these become $22.58 \mathrm{mag}, 23.06 \mathrm{mag}$, and $22.29 \mathrm{mag}$, respectively. The average of these is 4.3 mag brighter than the middle range for our H-band magnitudes of tadpoles, and this difference explains the factor of $\sim 100$ larger stellar masses in the Förster Schreiber et al. tadpoles. Evidently, tadpole structures are not limited to low-mass galaxies, although the UDF tadpoles tend to be low-mass.

The position-velocity diagrams from gas emission lines for SSA22a-MD41, Q2346-BX405, and Q2346-BX482 in Förster Schreiber et al. are fairly straight throughout both the head and the tail parts, suggesting solid body rotation or some other uniform gradient in the line-of-sight motion. Q2343-BX389 has a 2-component position-velocity distribution, with a straight part in the head and another straight part with a lower gradient in the tail. The two velocities in Q2343-BX389 join well in the middle, where the head meets the tail, so the components are not likely to be from two different galaxies on the same line of sight. Looking closer, two of the tadpoles with the nearly straight position-velocity distributions, SSA22aMD41 and Q2346-BX482, also have slight kinks where their tails meet their heads, although within each component, the position-velocity distributions are straight. Only Q2346-BX405 has a position-velocity orientation that is the same for the head and the tail. The 3 cases with velocity kinks could be signatures of complex dynamics involving galaxy interactions. but they could also arise from other forcings. 
The model for tadpoles implicitly assumed by Foster Schreiber et al. is that each system is a single rotating disk and the dynamics reflects the potential well from a common dark matter halo. There is some preference for this model. The dynamical mass is proportional to the square of the velocity extent multiplied by the first power of the spatial extent. For a nearly straight position-velocity distribution, taking only half of the extent by cutting out the tail leads to a dynamical mass for the head that is smaller than the total by $1 / 8$. This would mean that the dynamical masses in the head regions would be comparable to or less than the total stellar masses given above (recall that we determined the total-to-stellar mass ratios above, and the average is 5.5). Because the heads represent a significant fraction of the total luminosities (e.g., for our tadpoles, the heads represent $30 \%-50 \%$ of the total luminosity, from Figure 2), it is unlikely that the heads alone trace the stellar+dark matter masses over half the extent of the position-velocity distribution, and the tails trace an unbound tidal or stripped feature unconnected with dark matter. Only for Q2343-BX389, which has the strongly kinked position-velocity distribution with a much weaker velocity gradient in the tail, might the tail be dynamically isolated from the head's dark matter.

Law et al. (2009) include a galaxy in their OSIRIS survey, Q1700-BX710, that appears to be a tadpole from the HST/ACS image. The redshift, $z=2.29$, is comparable to that of our tadpoles. The diameter of the bright part, $2.2 \mathrm{kpc}$, is slightly larger than our diameters, as are the stellar brightness, $K_{S}$ (Vega) $=20.23 \mathrm{mag}$ and SED mass $M=4.4 \times 10^{10} M_{\odot}$. There is no regular velocity rotation in Q1700-BX710, as the kinematics appears to be dominated by random motions with $V_{\text {shear }} / \sigma=0.2$ and $\sigma=68 \pm 25 \mathrm{~km} \mathrm{~s}^{-1}$, calculated as the average over the dispersion for each pixel. Law et al. state that the tail has a lower velocity dispersion than the head and points to another galaxy $41 \mathrm{kpc}$ away at the same redshift. Unlike the tadpole galaxies in Förster Schreiber et al. (2009) and most of those in the present study, the tadpole in Law et al. shows good evidence for an interaction.

\subsection{A few Odd Cases and Possible Face-on Tadpoles}

Our survey of UDF tadpoles uncovered a few odd cases with suggestive environments. Figure 14 shows a UDF field of view with 3 nearly-aligned tadpoles and a double-galaxy. Figure 15 shows another field with 4 clumpy galaxies, two of which are tadpoles. These fields could be evidence for environment effects, such as ram pressure stripping in dense cosmological flows. However, recall that tadpoles do not have a statistically significant excess or deficit of other equally-large galaxies around them, nor do they have any preferred orientation relative to their companions, as discussed above. Therefore these two odd cases could be statistical flukes. 
The odd cases do suggest another interpretation for tadpoles, however. When combined with the generally irregular morphology of clumpy, high-redshift disks, and the common occurrence of double-clump galaxies like those in Figures 14 and 15, we get the impression that some disks might have only one big clump, and in the tadpole class, these single clumps are viewed at the projected edges of the disks. This model is also consistent with the kinematical data presented in the previous section (if it applies to our galaxies), which suggested that the full extent of the tadpole is required for the rotation curve to give a total dynamical mass reasonably larger than the SED-fitted stellar mass (i.e., to account for dark matter).

Figure [16] shows 5 examples of clumpy disks with single, lop-sided clumps. Some are ring-like (other examples of ring-like clump clusters are in Elmegreen et al. 2009a). The bottom right example is most likely an edge-on disk with a large star forming region at one end. It is something like a chain galaxy, but with only one clump in the chain. The lop-sided ring clump clusters may look like tadpoles when viewed edge-on.

A remote possibility is that the tails are the result of supernova-driven winds consisting of driven gas and the star formation in it, in addition to gravitationally entrained old stars. This is unlikely because winds leaving a galaxy (presumably the tadpole head) should be bipolar or more isotropic than the one-sided tail. It is also energetically unreasonable to gravitationally drag from the head the relatively high stellar masses that we measure in the tails.

\section{Discussion}

The tadpole galaxies in the UDF have relatively low-mass heads and tails, and they are generally young when viewed in ACS-band colors, which are in the restframe uv. The same is true when NICMOS colors are added. The tail mass is comparable to or slightly larger than the head mass, and the head ages are about the same as the tail ages, to within the uncertainties of our model fits. We cannot tell the origin of tadpoles from these measurements alone.

Tadpoles resemble poorly-resolved versions of tidally interacting galaxies, such as the namesake "Tadpole Galaxy," UGC 10214. Occasionally there is a double head to support this model (Figure 12), but this situation is rare (13\% for UDF tadpoles). Still, a plausible model for the origin of tadpole structure is a galaxy merger, even though the merger remnants

are not usually visible in the tadpole heads, the tadpole tails are straighter and more regular than most merger tails, and no excess of nearby large galaxies is systematically seen (Sect. 
4.2). A $z=2.29$ tadpole galaxy that could be a merger has a chaotic velocity field and a nearby companion in a study by Law et al. (2009) (Sect 4.3).

Tadpoles also resemble comets that suggest a wind-swept origin. They would have to be gas-rich in this case, especially the heads, to put so much star-forming mass in the sweptback tails. They would also have to be moving though a dense intergalactic medium for ram pressure to have much of an effect. Considering that the average stellar density of a tadpole head is $\sim 2 M_{\odot} \mathrm{pc}^{-3}$ (Sect. 4.1), which is $1.4 \times 10^{-22} \mathrm{gm} \mathrm{cm}^{-3}$, the corresponding density in hydrogen atoms, including helium, would be $\sim 70 \mathrm{~cm}^{-3}$. If the gas mass in the head is comparable to the stellar mass, as in other young galaxies (Tacconi et al. 2010), then the gas density would be about the same. In order to unbind a lot of this gas and move it into a tail, the intergalactic density would have to be comparable to the head density if the tadpole moved through this medium at about its own escape velocity. This velocity is $\sim 40 \mathrm{~km} \mathrm{~s}^{-1}$ from the stellar mass and radius alone, and probably $3 \times$ larger if there is $10 \times$ the stellar mass in dark matter. For larger tadpole velocities, $v$, the intergalactic density could be less by $1 / v^{2}$ and have the same ram pressure.

Intergalactic ram pressure is observed locally to produce a morphology similar to what we see in tadpoles. Chung et al. (2009) studied the HI and stellar distributions in Virgo Cluster galaxies. IC 3418 (their Fig. 2) is an IBm galaxy with a uv-bright trail of stars extending $9 \mathrm{kpc}$ southeast of its main body. There is no detectable gas now but the authors suggest that the tail formerly contained gas that was converted into stars by the cluster pressure. Other Virgo spirals have slightly displaced gas disks or truncated gas disks as a result of the ram pressure from their motion through the hot intergalactic gas. NGC 4294 in Virgo has a $27 \mathrm{kpc}$ long tail of HI without any stars down to $26 \mathrm{mag} \operatorname{arcsec}^{2}$. Its neighbor, NGC 4299, has a similar starless HI tail parallel to the one in NGC 4294. These tails are probably from ram pressure, but the two galaxies also show signs of an interaction.

For the tadpoles in the UDF, the tails are much more massive in young stars than they are for the tadpole in Virgo. The intergalactic density should be larger for the UDF tadpoles if there are active cosmological gas inflows to nearby galaxies. The UDF tadpoles could be going through these dense flows. The velocity of the UDF tadpoles through the intergalactic medium should be smaller than the infall velocities in Virgo because the Virgo potential well is deep now; massive galaxy clusters were not well developed at $z \sim 2$. Whether the ram pressure was greater for the UDF tadpoles than the Virgo tadpole cannot be determined yet, but even at the same ram pressure, the high intergalactic density in the UDF should have a strong gas-stripping effect on the tadpoles. Numerical simulations of young galaxies moving through cosmological flows could test this. If a low mass galaxy with a high gas fraction moves through a dense, cold-flow region, then we predict a large fraction of the gas will get 
drawn into a tail. Star formation should occur in this tail, and because the gas fraction is high, a significant fraction of old stars in the original galaxy could get drawn into the tail as well.

Tadpoles could also be edge-on disks with a single, large, star-forming clump. If the clump is near the center, we might say the galaxy is normal, edge-on, and has a bulge, or we might call it a chain galaxy if the clump is very blue. When the clump is near the edge of the disk, however, it should look like a tadpole when viewed edge-on from the right orientation. Some examples of lop-sided, single-clump galaxies were shown in Section 4.4. This third model for tadpoles suggests they are single disks with a common halo of dark matter. This

is the most logical explanation for the three tadpoles studied by Förster Schreiber et al. (2009) that have position-velocity distributions with near-constant velocity gradients.

\section{Conclusions}

Tadpole galaxies as a morphological class could be a mixture of several types: (1) mergers, which should show double-heads if resolved properly, chaotic internal motions, and tails with peculiar velocities relative to the head rotation curves, (2) ram-pressure stripped heads and their debris tails that are interacting with a dense intergalactic gas or cosmological gas flow, (3) ram-pressure induced star formation at the edge of a low surface brightness galaxy disk, viewed edge-on, and (4) naturally lop-sided clumpy disks that are viewed edgeon.

Tadpole heads in the UDF have photometric masses from the rest-frame uv and visible passbands that are in the range of $10^{6}-10^{9} M_{\odot}$, increasing with redshift because of selection effects on size, surface brightness, and rest wavelength. The masses are comparable to the masses of bulge-like objects in other UDF clumpy galaxies, and also comparable to the largest clumps in clump-clusters and chains. Head ages span a wide range around and below 1 Gyr, but are poorly constrained by the SEDs considering the uncertainty with internal extinction.

Acknowledgmenta: We are grateful to the referee for helpful comments. DME thanks Vassar College for support from the astronomy publication fund.

\section{REFERENCES}

Abraham, R.G., van den Bergh, S., Glazebrook, K., Ellis, R.S., Santiago, B.X., Surma, P., \& Griffiths, R.E. 1996, ApJS, 107, 1 
Agertz, O., Teyssier, R., Moore, B. 2009, MNRAS, 397, L64

Beckwith, S.V.W., et al. 2006, AJ, 132, 1729

Bournaud, F., \& Elmegreen, B.G. 2009, ApJL, 694, 158

Bournaud, F., Elmegreen, B.G., \& Martig, M. 2009, ApJL, 707, 1

Brooks, A. M., Governato, F., Quinn, T., Brook, C. B., \& Wadsley, J. 2009, ApJ, 694, 396

Bruzual, G. \& Charlot, S. 2003, MNRAS, 344, 1000

Calzetti, D., Armus, L., Bohlin, R.C., Kinney, A.L., Koornneef, J., \& Storchi-Bergmann, T. 2000, ApJ, 533, 682

Chung, A., van Gorkom, J.H., Kenney, J.D.P., Crowl, H., \& Vollmer, B. 2009, AJ, 138, 1741

Coe, D., Benítez, N., Sanchez, F., Jee, M., Bouwens, R., \& Ford, H. 2006, AJ, 132, 926

Conselice, C.J., \& Arnold, J. 2009, MNRAS, 397, 208

Dekel, A., Sari, R., \& Ceverino, D. 2009, ApJ 703, 785

De Mello et al. 2006a, AJ, 131, 216

De Mello et al. 2006b, AJ, 132, 2014

Elmegreen, D.M., Elmegreen, B.G., Rubin, D.S., and Schaffer, M.A. 2005, ApJ, 631, 85

Elmegreen, D.M., Elmegreen, B.G., Ravindranath, S., Coe, D.A. 2007, ApJ, 658, 763

Elmegreen, B.G., Elmegreen, D.M., Fernandez, M.X., \& Lemonias, J.J., 2009a, ApJ, 692, 12

Elmegreen, D.M., Elmegreen, B.G., Marcus, M., Shahinyan, K., Yau, M., \& Petersen, M. 2009b, ApJ, 701, 306

Förster Schreiber, et al. 2009, ApJ, 706, 1364

Giavalisco, M., et al. 2004, ApJ, 600, L93

Kereŝ, D., Katz, N., Fardal, M., Davé, R., \& Weinberg, D. H. 2009, MNRAS, 395, 160

Law, D.R., Steidel, C.C., Erb, D.K., Larkin, J.E., Pettini, M., Shapley, A.E., Wright, S.A. 2009, ApJ, 697, 2057

Leitherer, C., Li, I.-H., Calzetti, D., Heckman, T.M. 2002, ApJS, 140, 303 
Madau, P. 1995, ApJ, 441, 18

Ocvirk, P., Pichon, C., \& Teyssier, R. 2008, MNRAS, 390, 1326

Rafelski, M., Wolfe, A.M., Cooke, J., Chen, H.-W., Armandroff, T.E., \& Wirth, G.D. 2009, ApJ, 703, 2033

Rawat, A., Kembhavi, A.K., Hammer, F., Flores, H., \& Barway, S. 2007, A\&A, 469, 483

Rhoads, J.E., et al. 2005, ApJ, 621, 582

Spergel, D.N., et al. 2003, ApJS, 148, 175

Springel, V., Di Matteo, T., \& Hernquist, L, 2005a, ApJL, 620, L79

Springel, V., Di Matteo, T., \& Hernquist, L, 2005b, MNRAS, 361, 776

Stanway, E.R., McMahon, R.G., \& Bunker, A.J. 2005, MNRAS, 359, 1184

Straughn, A. N., Cohen, S. H., Ryan, R. E., Hathi, N. P., Windhorst, R. A., \& Jansen, R. A. 2006, ApJ, 639, 724

Tacconi, L., et al. 2010, Nature, 463, 781

Thompson, R. I., et al. 2005, AJ, 130, 1

van den Bergh, S., Abraham, R.G., Ellis, R.S., Tanvir, N.R., Santiago, B.X., \& Glazebrook, K.G. 1996, AJ 112, 359

Windhorst, R.A., et al. 2006, New Astron., 50, 821 

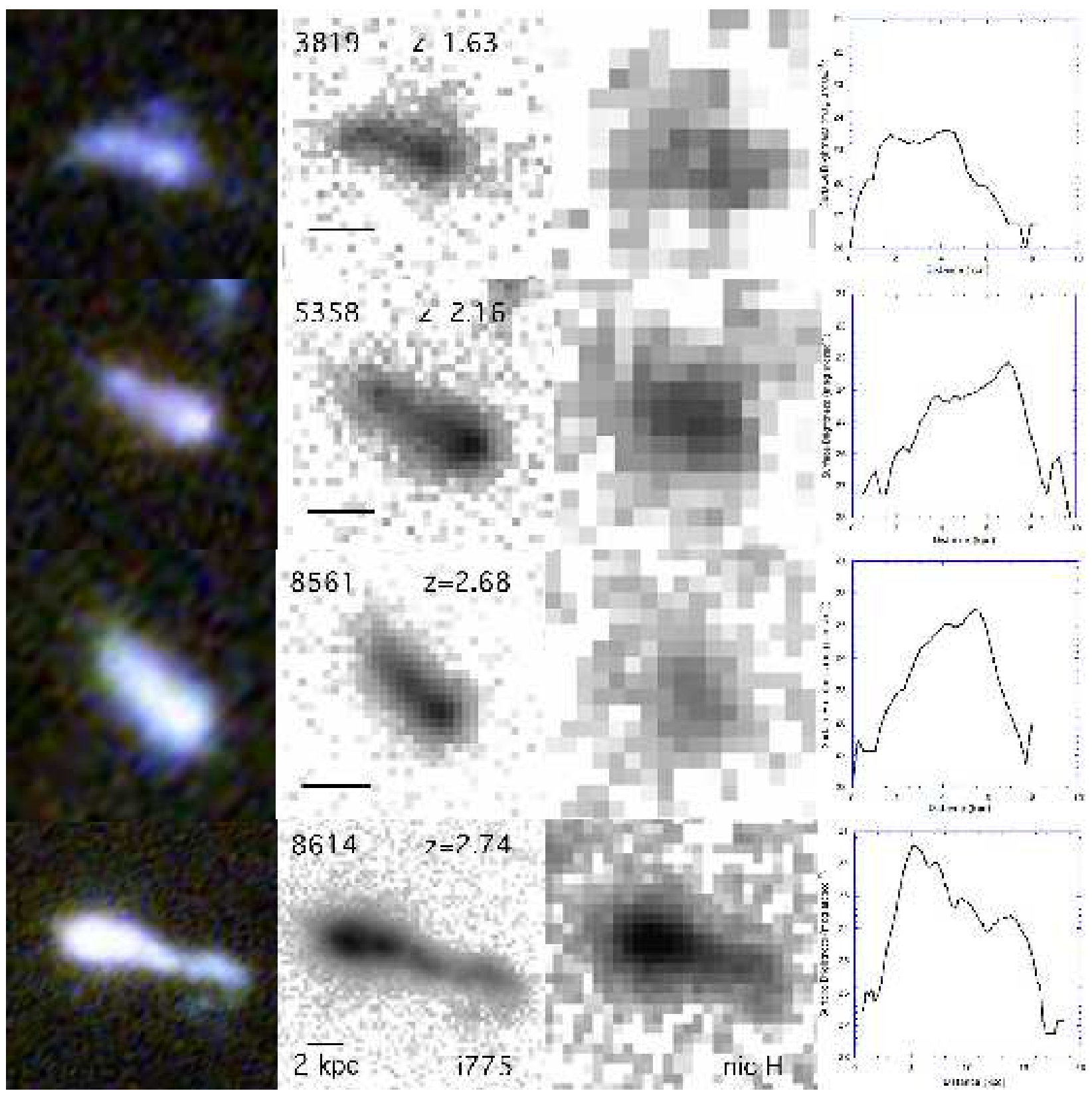

Fig. 1.- Four tadpole galaxies from the Hubble UDF, with color Sky Walker images from the ACS filters on the left, $i_{775}$ next, NICMOS H-band next, and an intensity scan through the length of the galaxy in $i_{775}$ on the right. UDF 8614 on the bottom has a double-core head with the two cores aligned with the tail. [Image quality degraded for arXiv] 


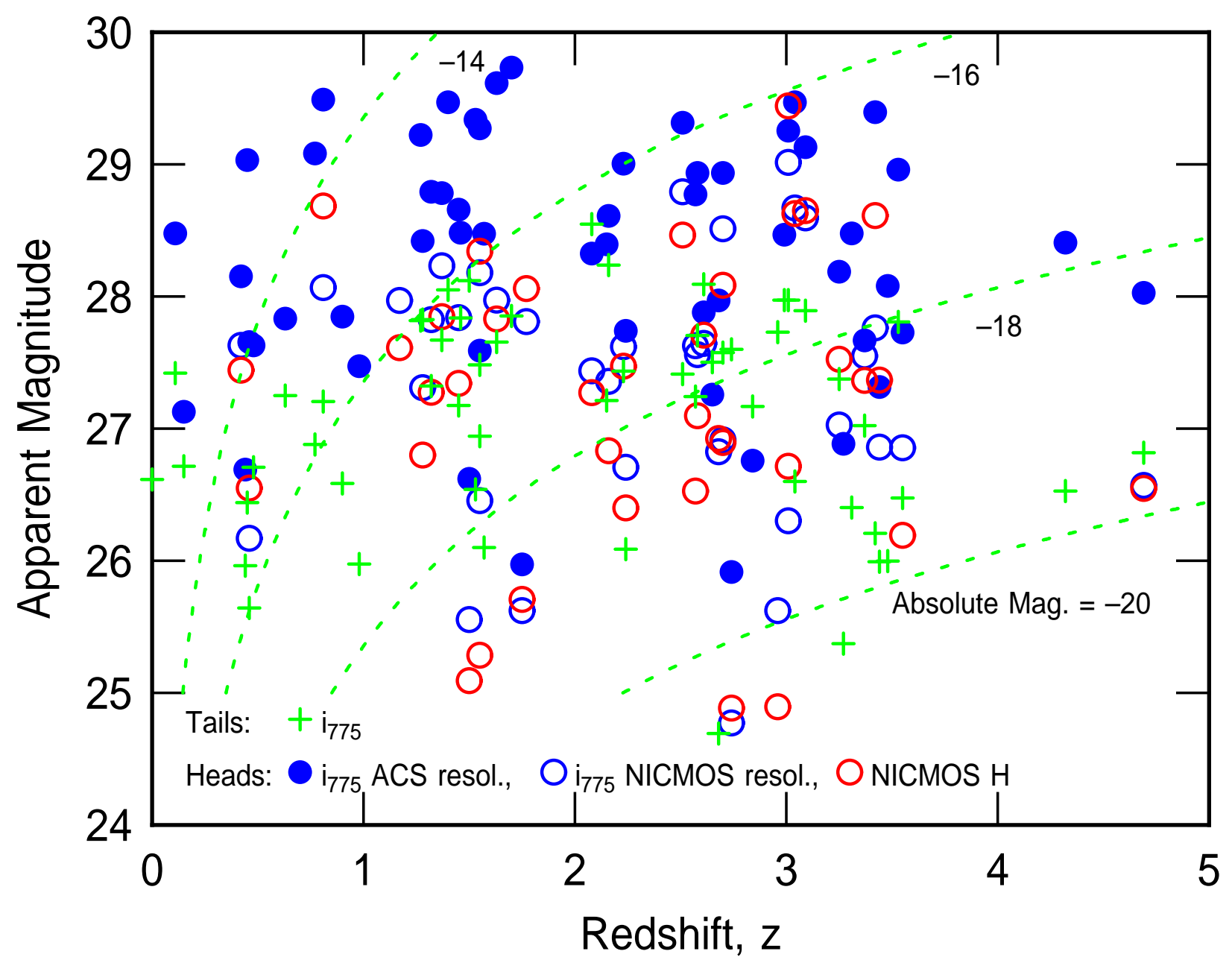

Fig. 2.- Magnitudes of the heads (dots) and tails (plus symbols) at ACS resolution in $i_{775}$ band, $i_{775}$ band magnitudes of the heads at the NICMOS resolution (blue circles), and NICMOS H-band magnitudes of the heads (red circles). The dotted lines show absolute magnitudes, as indicated. 


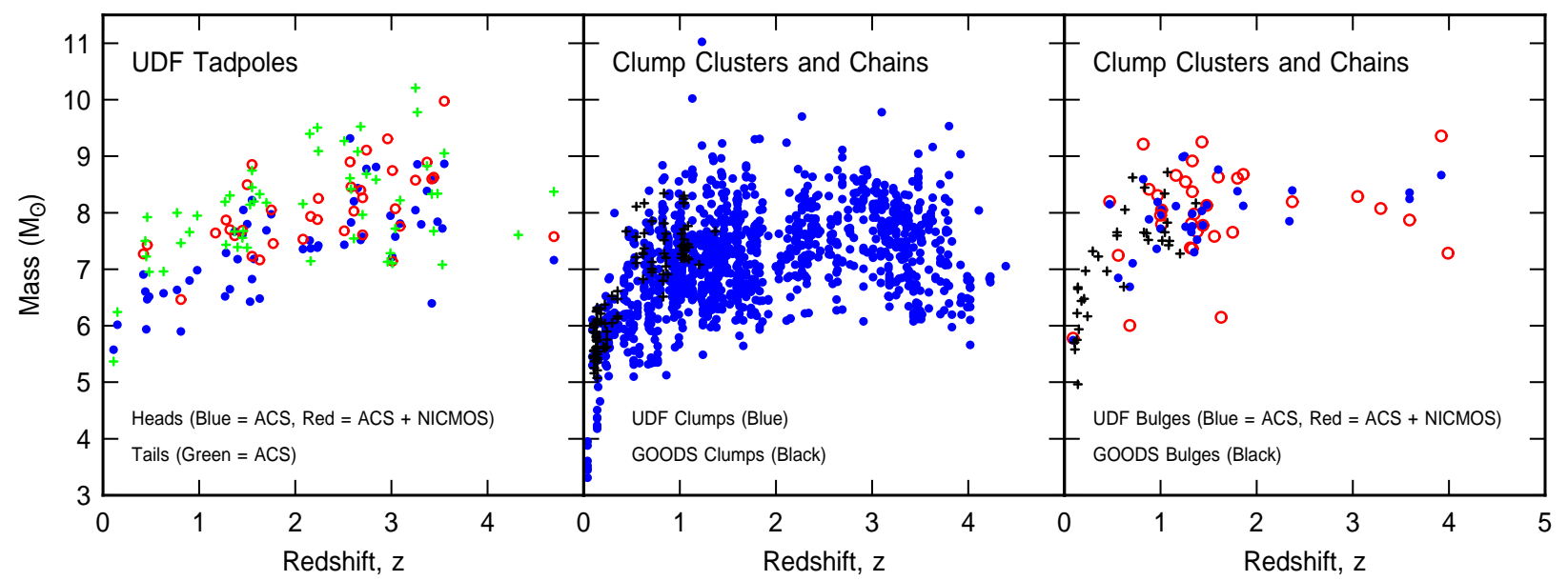

Fig. 3.- (left) Masses of the tadpole heads and tails versus redshift. Tail masses (green plus symbols) used only the full resolution ACS bands. Head masses were evaluated twice, once with ACS-only filters at full resolution (blue dots), and again with NICMOS filters combined with ACS filters blurred to NICMOS resolution (red circles). (middle) Masses of clumps in clump cluster and chains galaxies in the UDF (blue dots) and GOODS fields (black). (right) Masses of bulges or bulge-like objects in clump clusters and chains in the UDF (blue dots with ACS resolution; red circles with NICMOS filters and ACS filters at NICMOS resolution), and GOODS (black). The clump cluster and chain measurements are from Elmegreen et al. (2009a,, b) . 


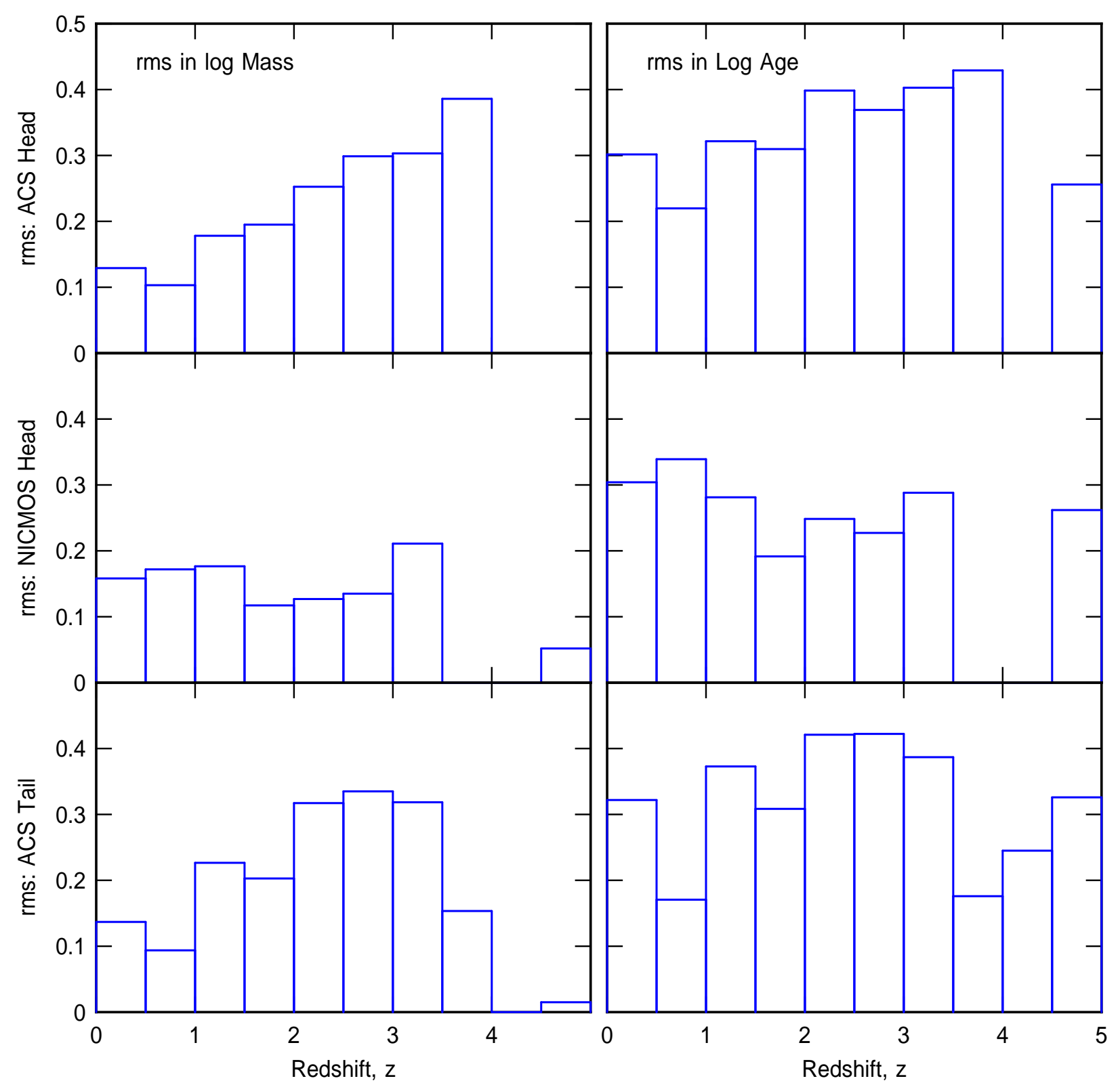

Fig. 4.- (left) The rms deviations in the log of the measured masses are shown, averaged over bins of 0.5 in redshift. The top, middle and bottom panels are for ACS measurements of the heads, ACS+NICMOS measurement of the heads, and ACS measurements of the tails. The masses are calculated in $M_{\odot}$. For the ACS+NICMOS measurements, the $\log M$ of the heads is accurate to about \pm 0.15 , which corresponds to a factor of 1.4. (right) The rms deviations in the log of the ages for the same three measurement cases. Age rms values are higher than mass rms values because reddening effects from age and extinction partially cancel in the determination of mass. 


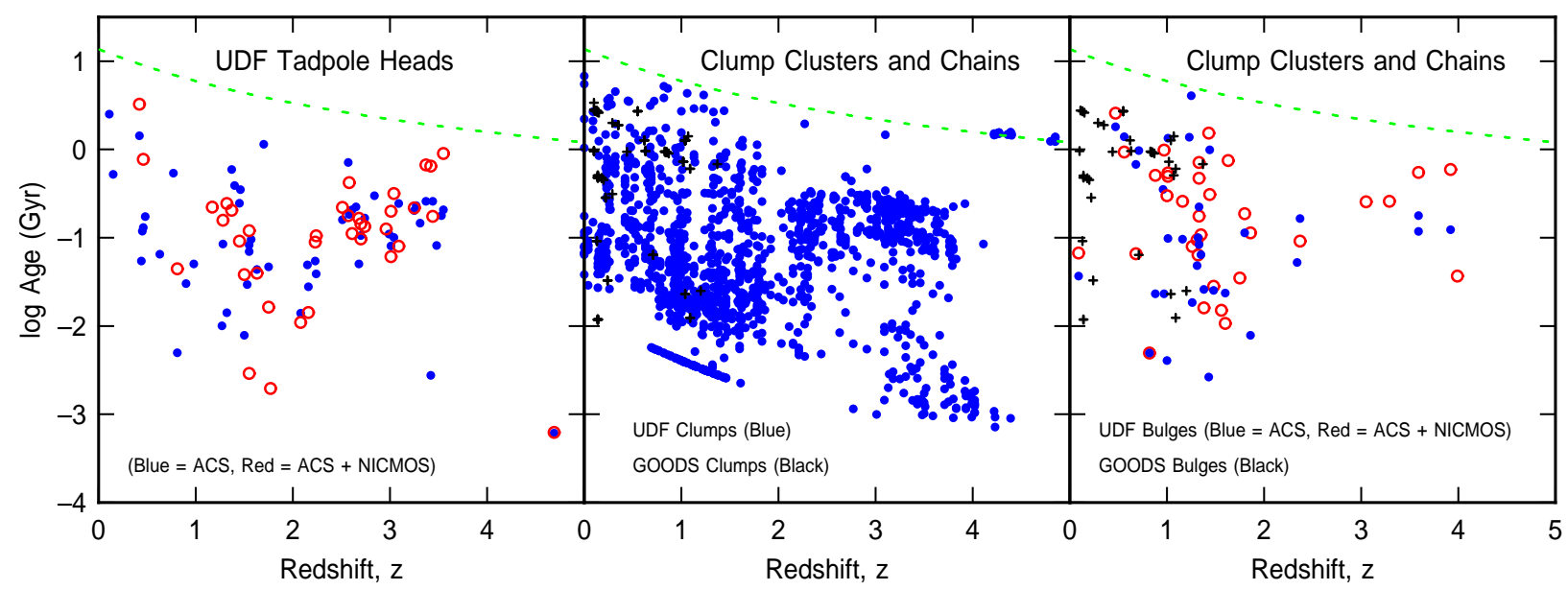

Fig. 5.- (left) Ages of the tadpole heads versus redshift. ACS-only filters are blue dots, and NICMOS filters combined with ACS filters blurred to NICMOS resolution are red circles. (middle) Ages of clumps in clump cluster and chains galaxies from the UDF (blue dots) and GOODS fields (black). (right) Ages of bulges or bulge-like objects in clump clusters and chains in the UDF (blue dots with ACS resolution; red circles with NICMOS filters and ACS filters at NICMOS resolution), and GOODS (black). The clump cluster and chain measurements are from Elmegreen et al. $(2009 \mathrm{a}, \mathrm{b})$. The green dashed lines represent the age of the universe as a function of redshift. 


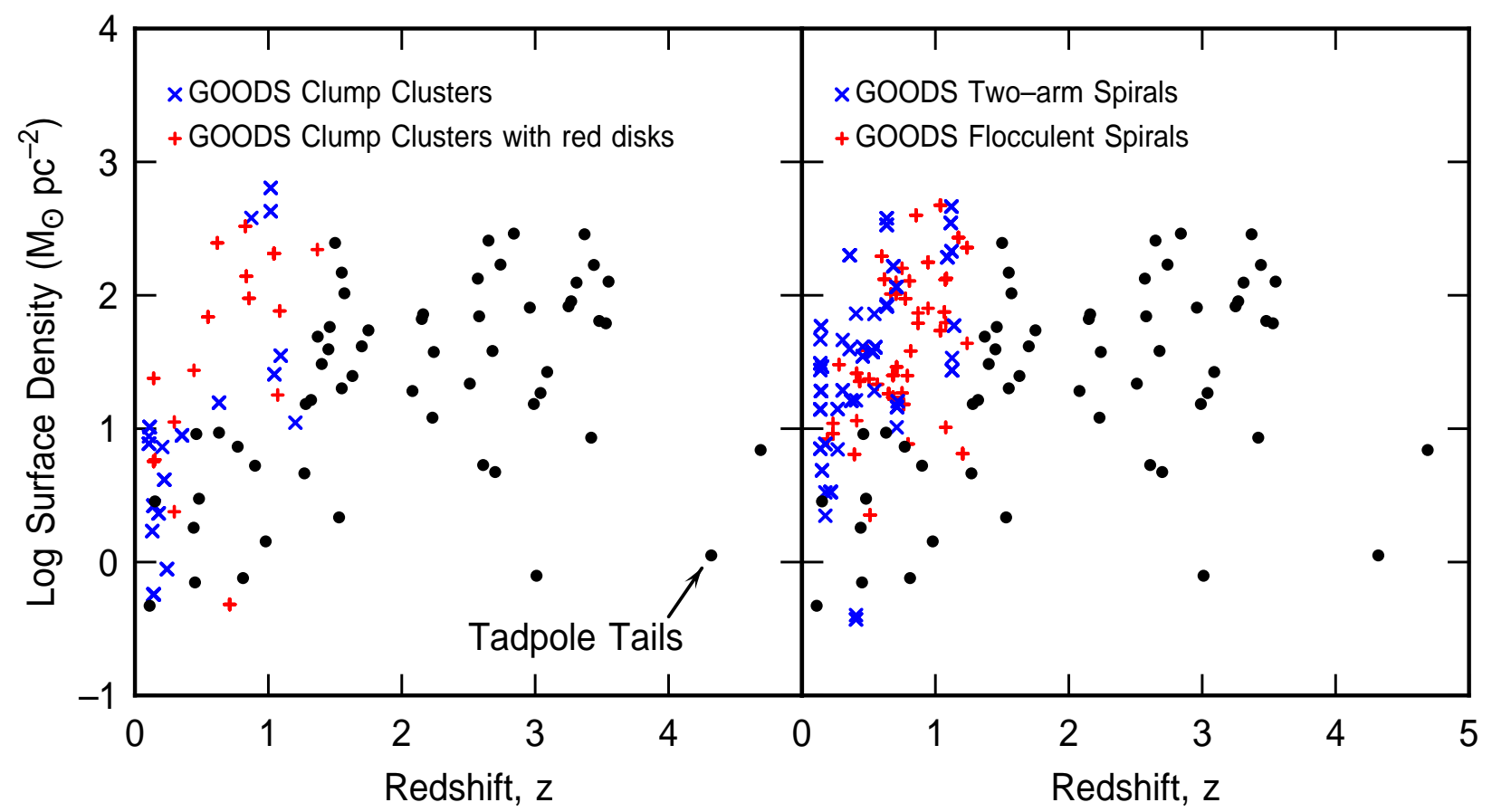

Fig. 6. - Mass surface densities of the tadpole tails (black dots, repeated in both panels) compared, on the left, to the interclump surface densities in GOODS clump clusters without red underlying disks (blue x-symbols) and with red underlying disks (red plus symbols). On the right, the tadpole tail surface densities are compared to the interclump surface densities in GOODS flocculent and grand design spirals. 


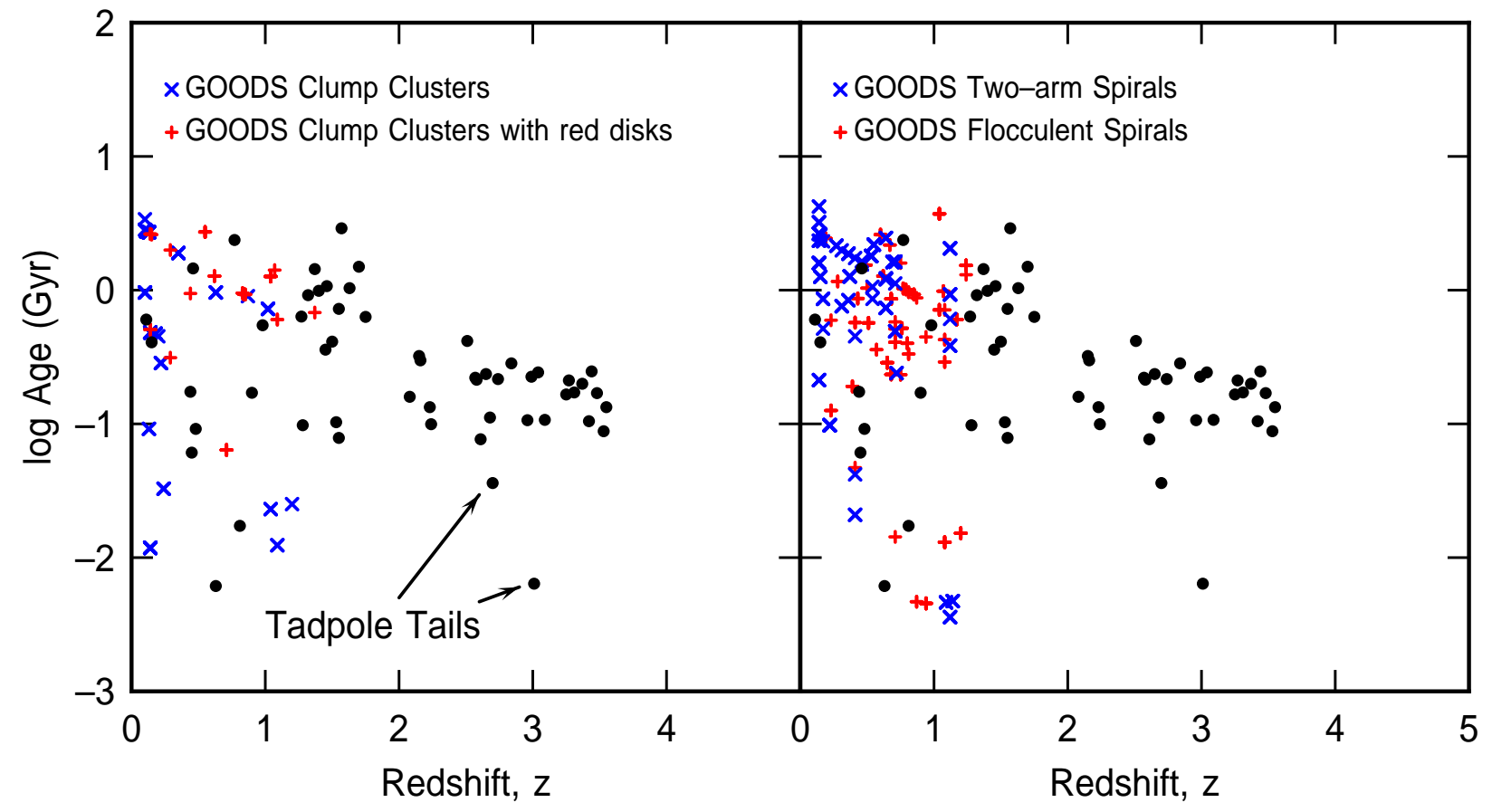

Fig. 7.- Ages of the tadpole tails (black dots, repeated in both panels) compared, on the left, to the interclump ages in GOODS clump clusters without red underlying disks (blue $\mathrm{x}$-symbols) and with red underlying disks (red plus symbols). On the right, the tadpole tail ages are compared to the interclump ages in GOODS flocculent and grand design spirals. 


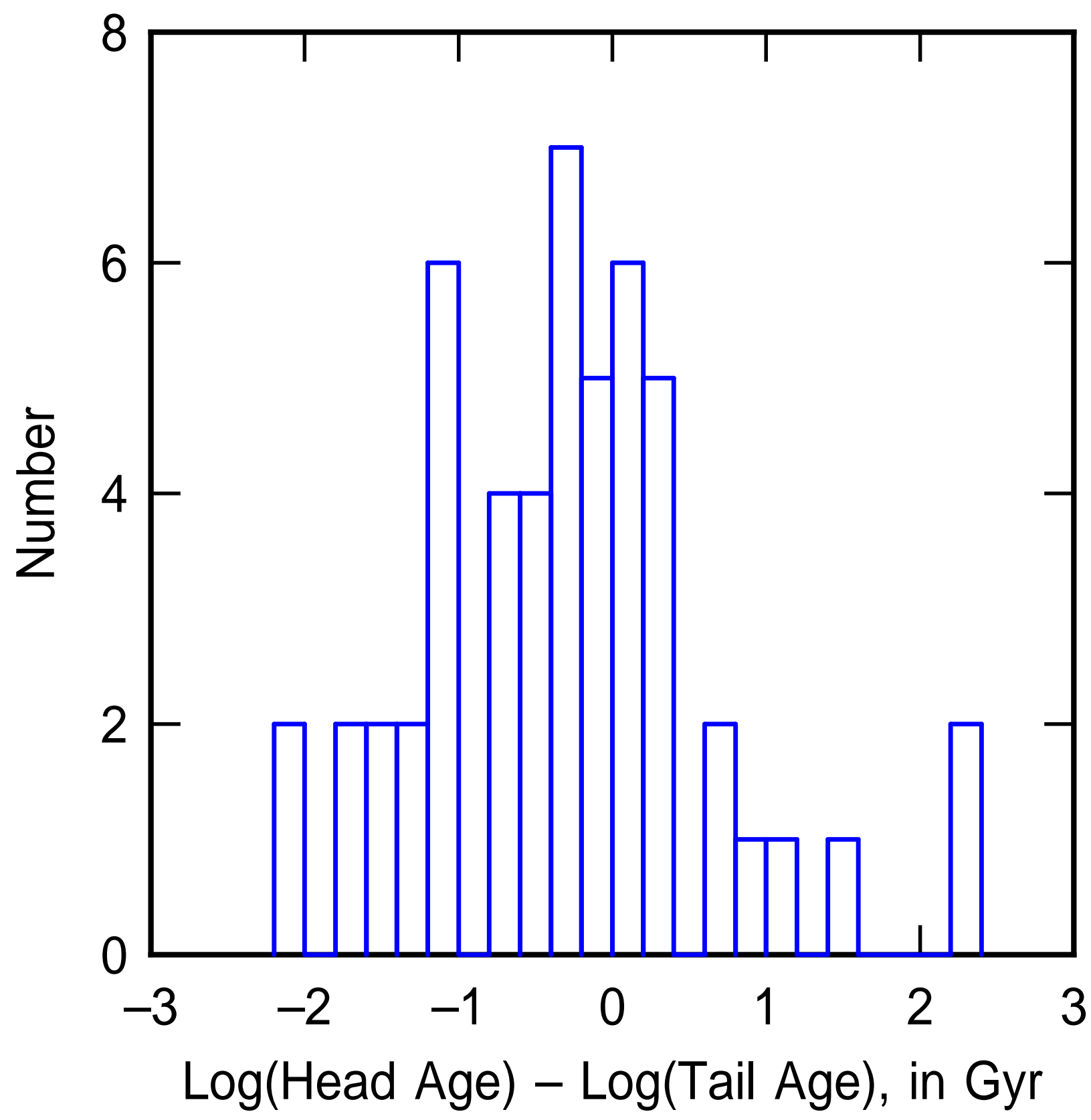

Fig. 8. - The distribution of the differences between the logs of the head and tail ages for individual tadpole galaxies. The average is about zero, meaning that the two components have about the same age on average. 


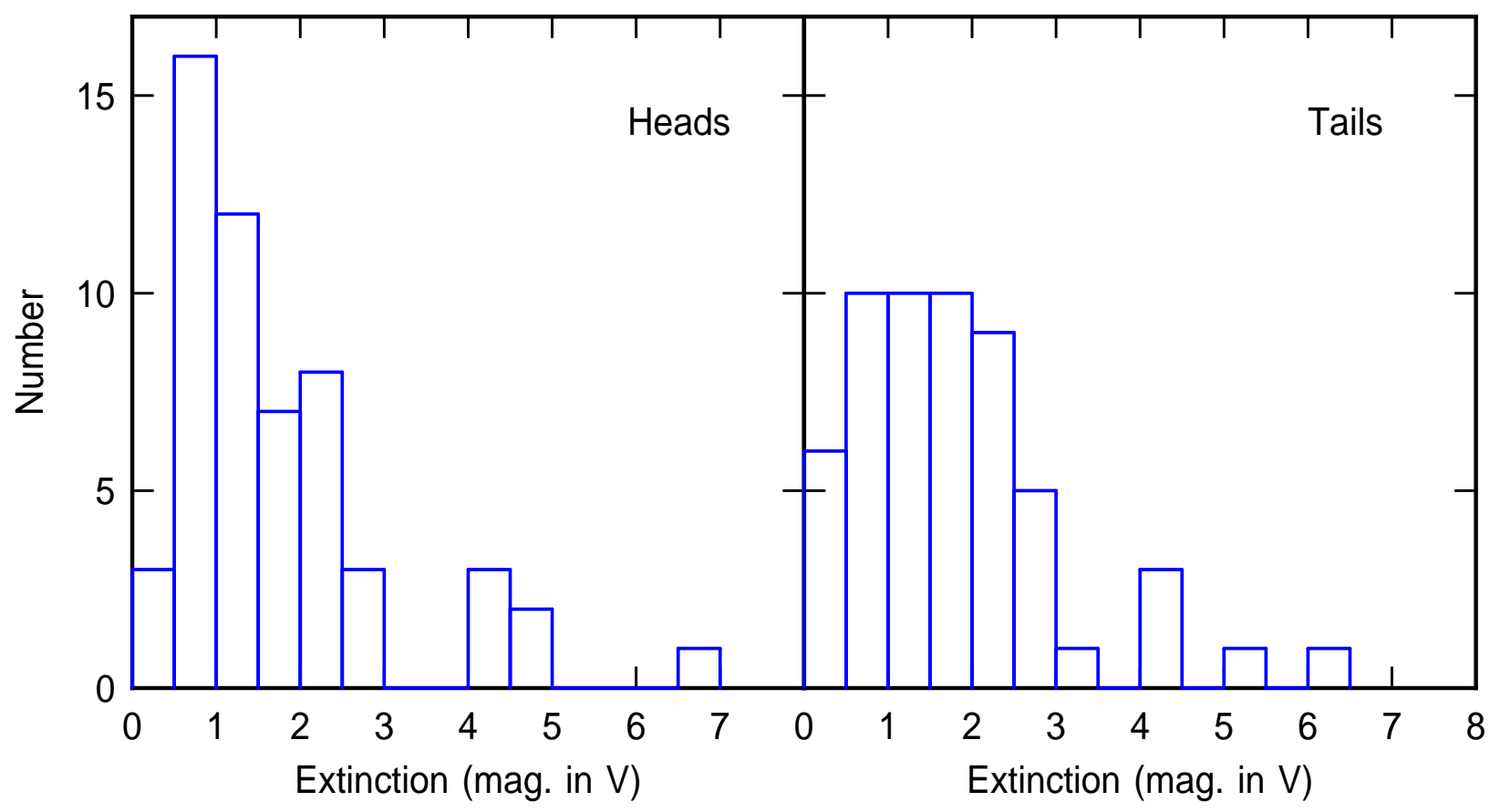

Fig. 9.- The distributions of the extinctions in V-band for the tadpole heads and tails, obtained as part of the fits to mass and age. 

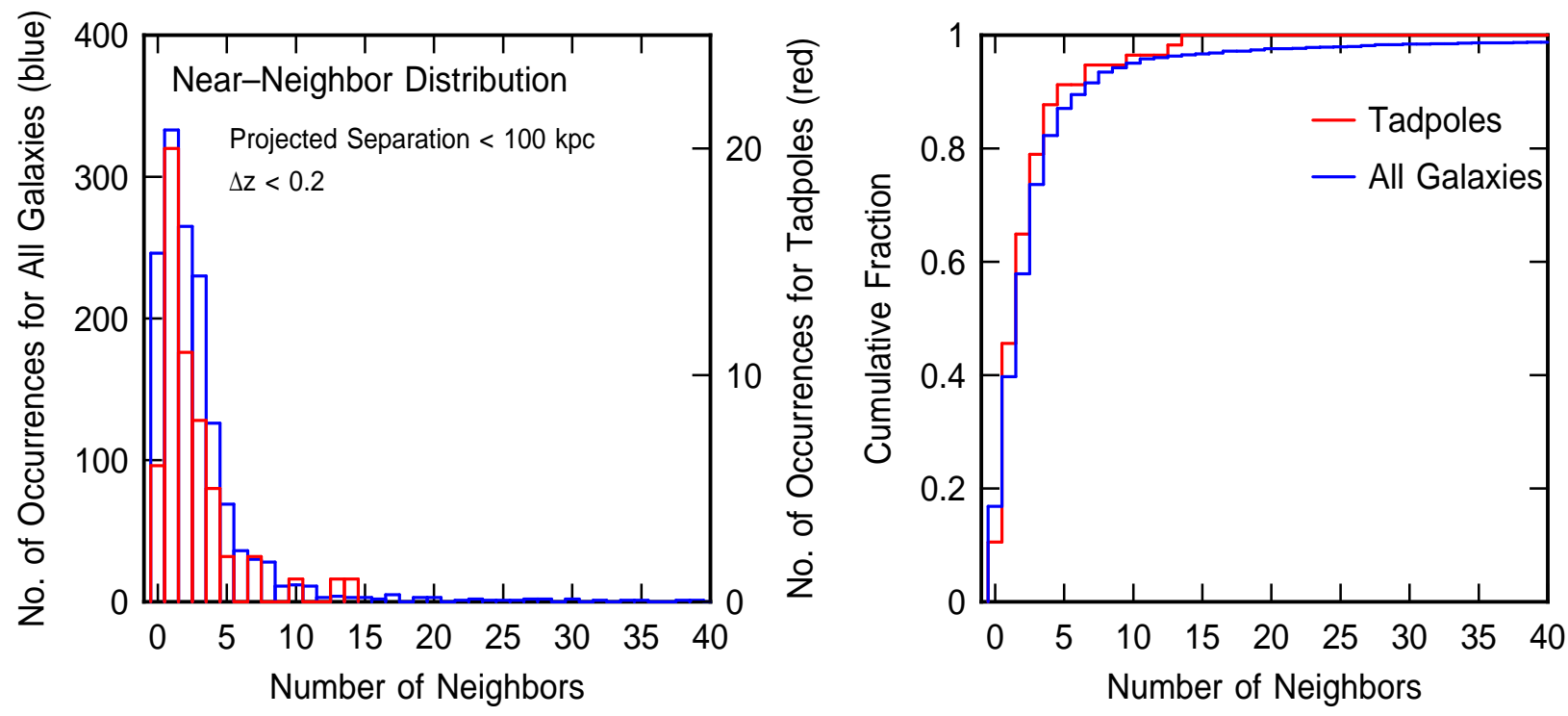

Fig. 10.- (left) The distribution function of companion galaxy counts within $100 \mathrm{kpc}$ projected distance from the tadpoles (red histogram, right-hand axis), and within a redshift interval of $\Delta z= \pm 0.2$. A companion is defined to be a galaxy in the UDF with a redshift in Rafelski et al. (2009) and an area larger than $100 \mathrm{px}^{2}$. The left-hand axis (blue histogram) shows the same companion distribution with respect to all galaxies larger than $100 \mathrm{px}^{2}$ in the same redshift catalog. The two distributions are about the same. (right) Normalized cumulative distributions obtained by integrating the histograms on the left. This figure, and a Kolmogorov-Smirnov test based on this figure, suggest that tadpole galaxies do not have an excess or deficit of neighbor galaxies with similar or larger sizes. 

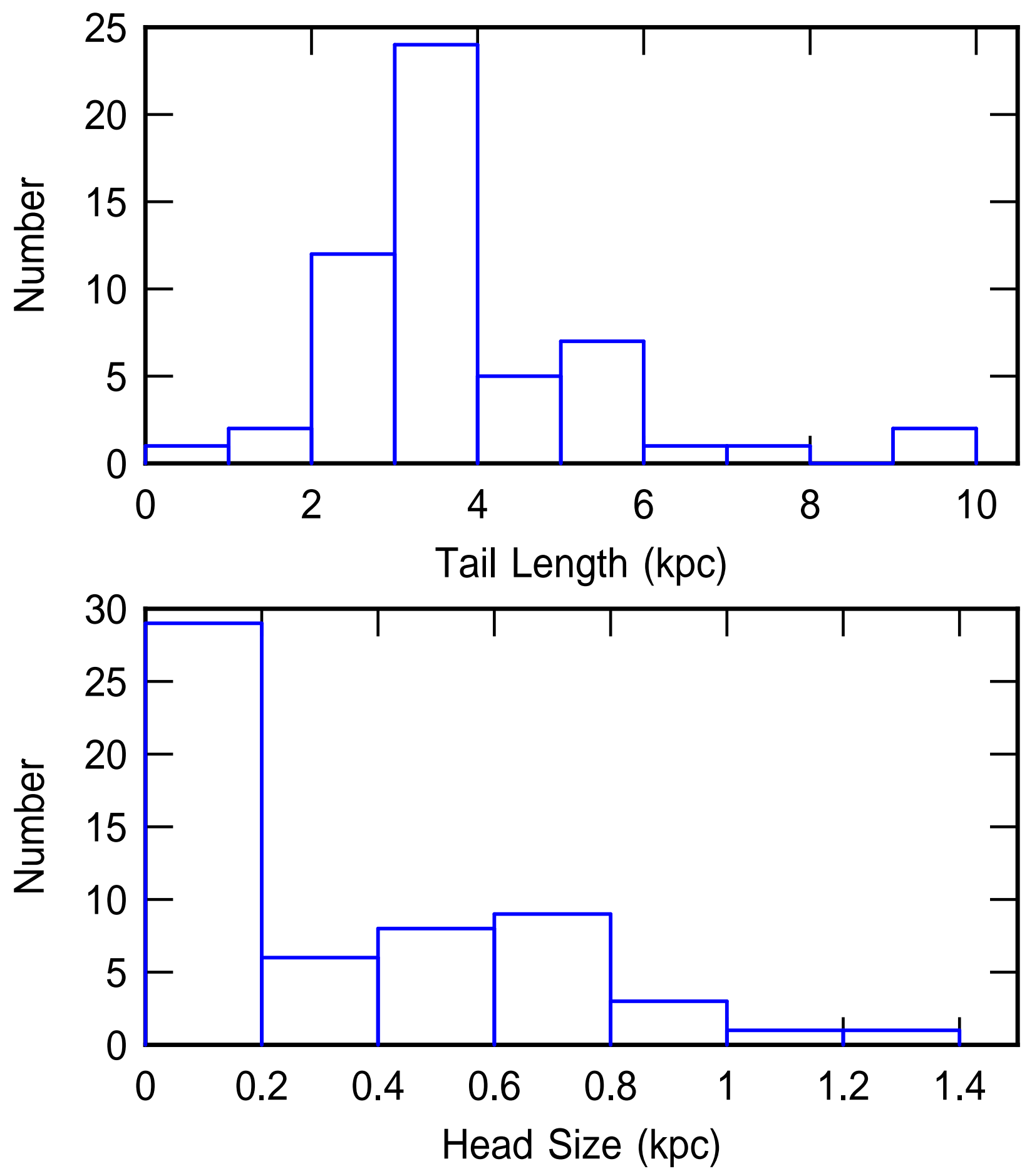

Fig. 11. - The distribution of tadpole head and tail sizes, measured as the square roots of the areas, considering the conversion from angular size to physical size at the redshift of the galaxy and correcting for point spread function. 


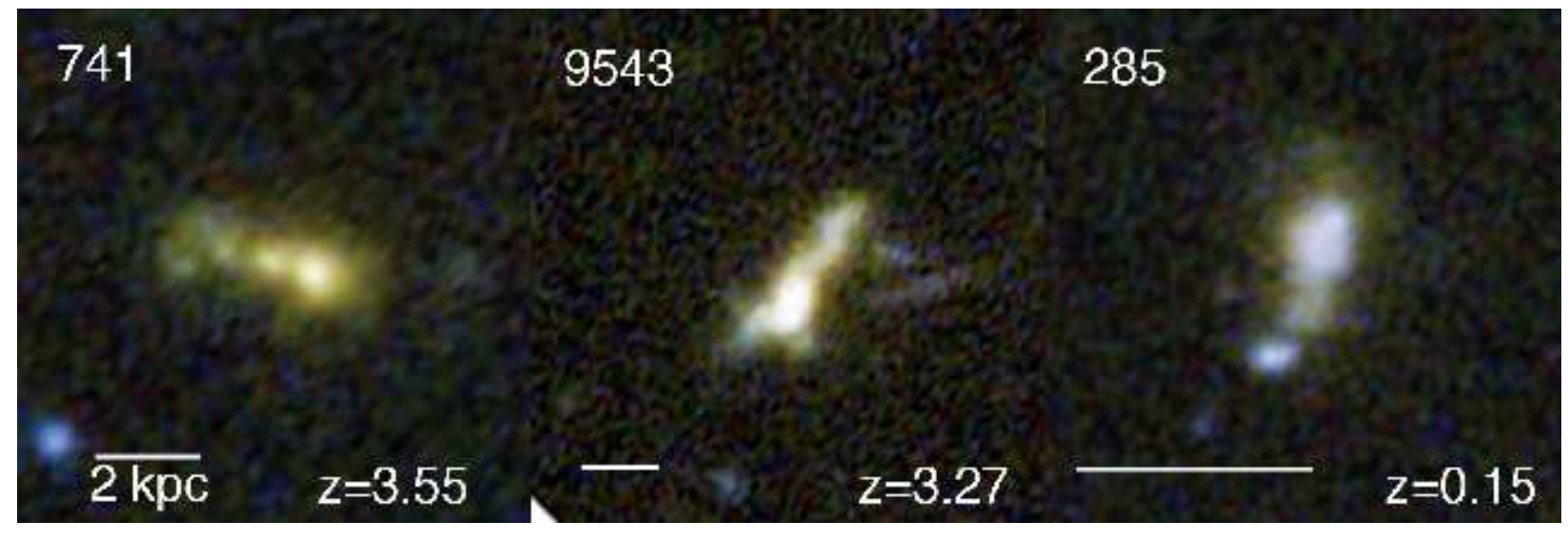

Fig. 12. - Three examples of tadpoles with clumpy structure that might indicate interactions between the clumps. Most tadpoles show single-core heads, suggesting they are not earlystage mergers. [Image quality degraded for arXiv] 


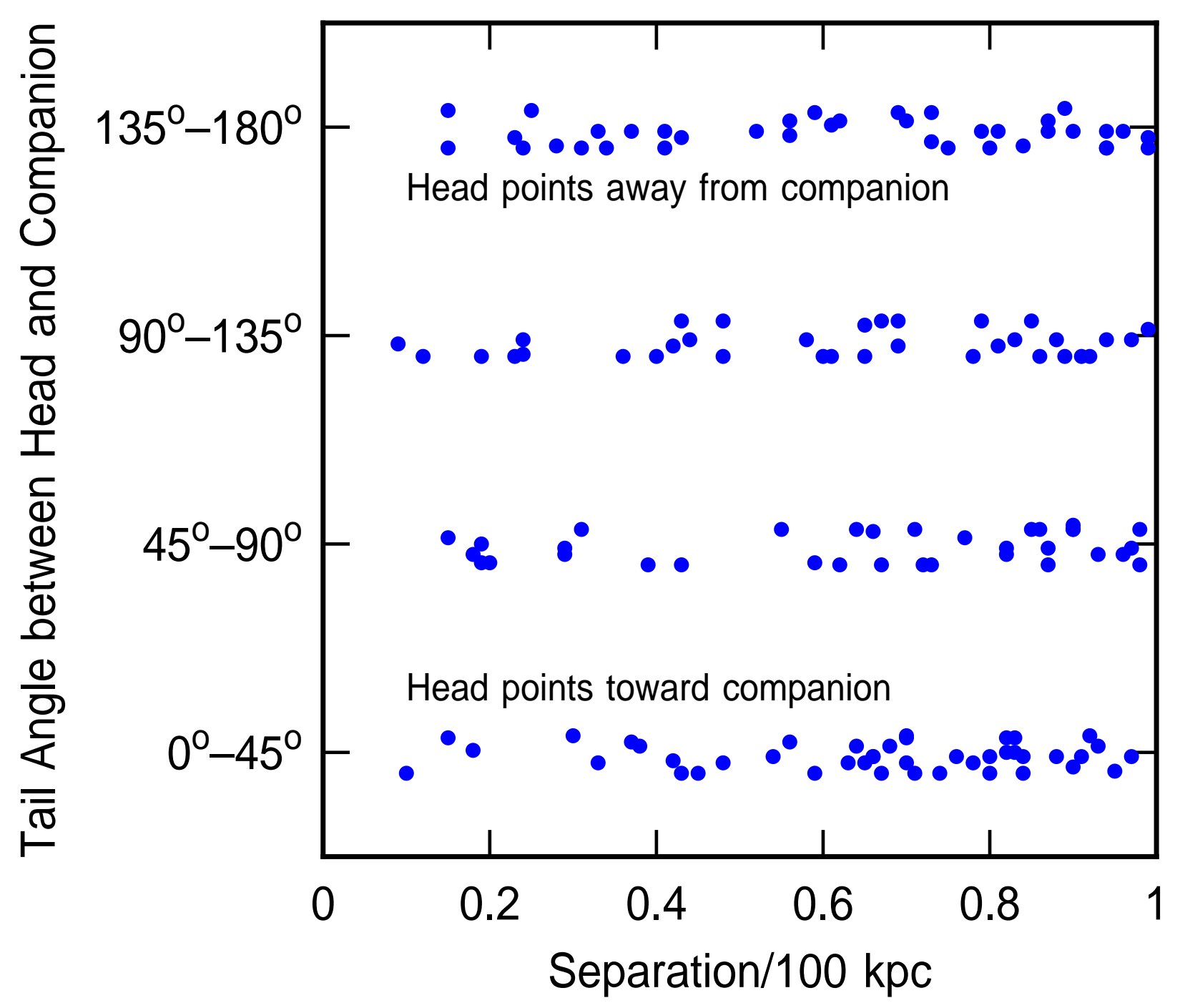

Fig. 13.- Distribution of the angle measured at the tail, between the head and a companion, for all companions within $100 \mathrm{kpc}$ projected separation and within a redshift interval of $\Delta z=0.2$. Each dot is a different combination of tadpole and companion galaxy. The convention for angle is that $0-45^{\circ}$ means the head is pointing toward the companion. 

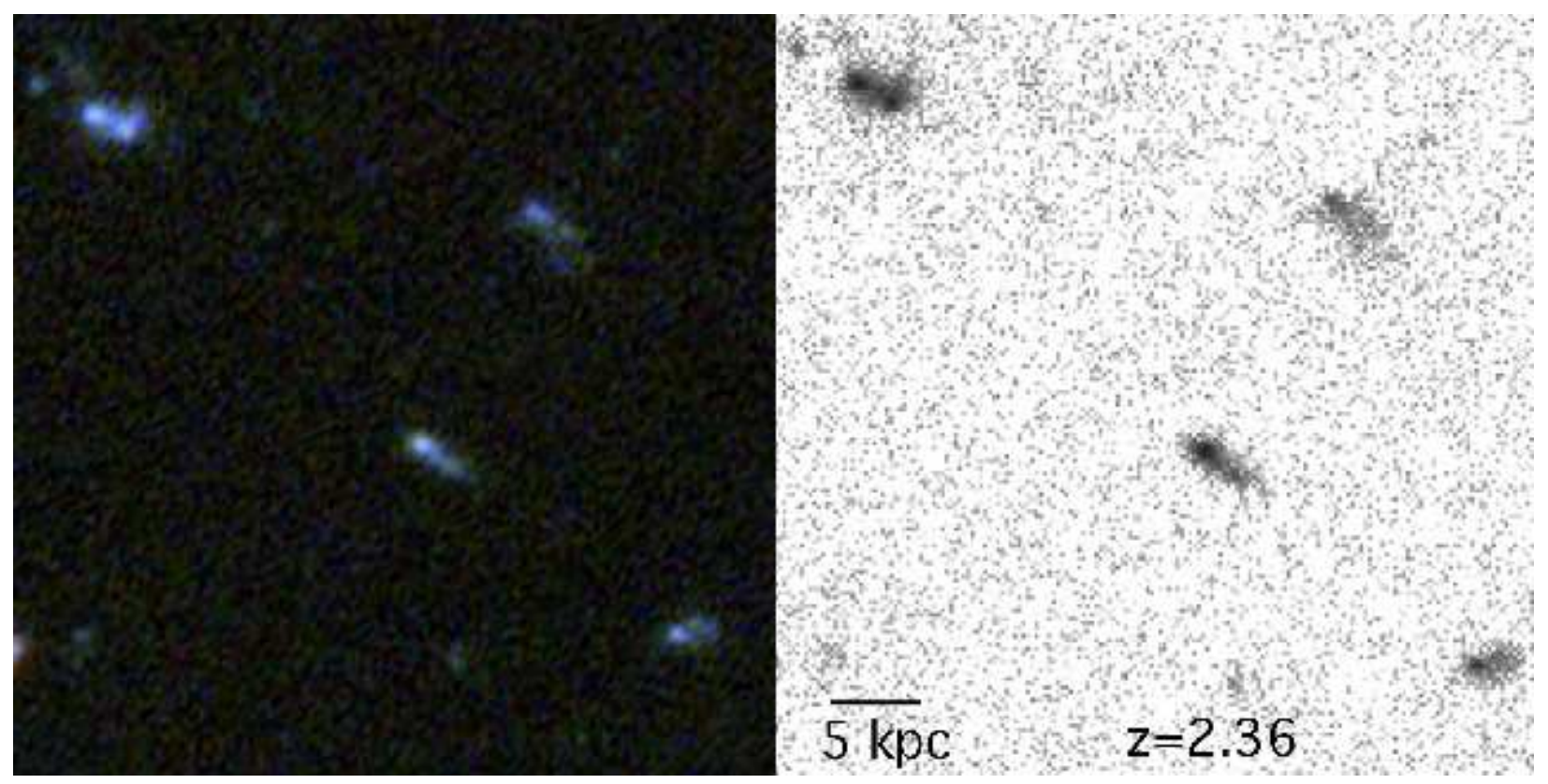

Fig. 14.- Four similar galaxies in a UDF field. The double-clump at the top left is UDF 4699, the tadpole at the top right is UDF 4682, the tadpole in the center is UDF 4592, and the tadpole at the lower right is UDF 4518. The redshifts using the method of Coe et al. (2006) as compiled in Elmegreen et al. (2007), are 1.96, 1.72, 2.36, and 2.49. Perhaps the galaxies are closer together than these photometric redshifts suggest. Redshifts for only two of these galaxies are in Rafelski et al. (2009): 2.34 for UDF 4699 and 2.7 for UDF 4592. [Image quality degraded for arXiv] 

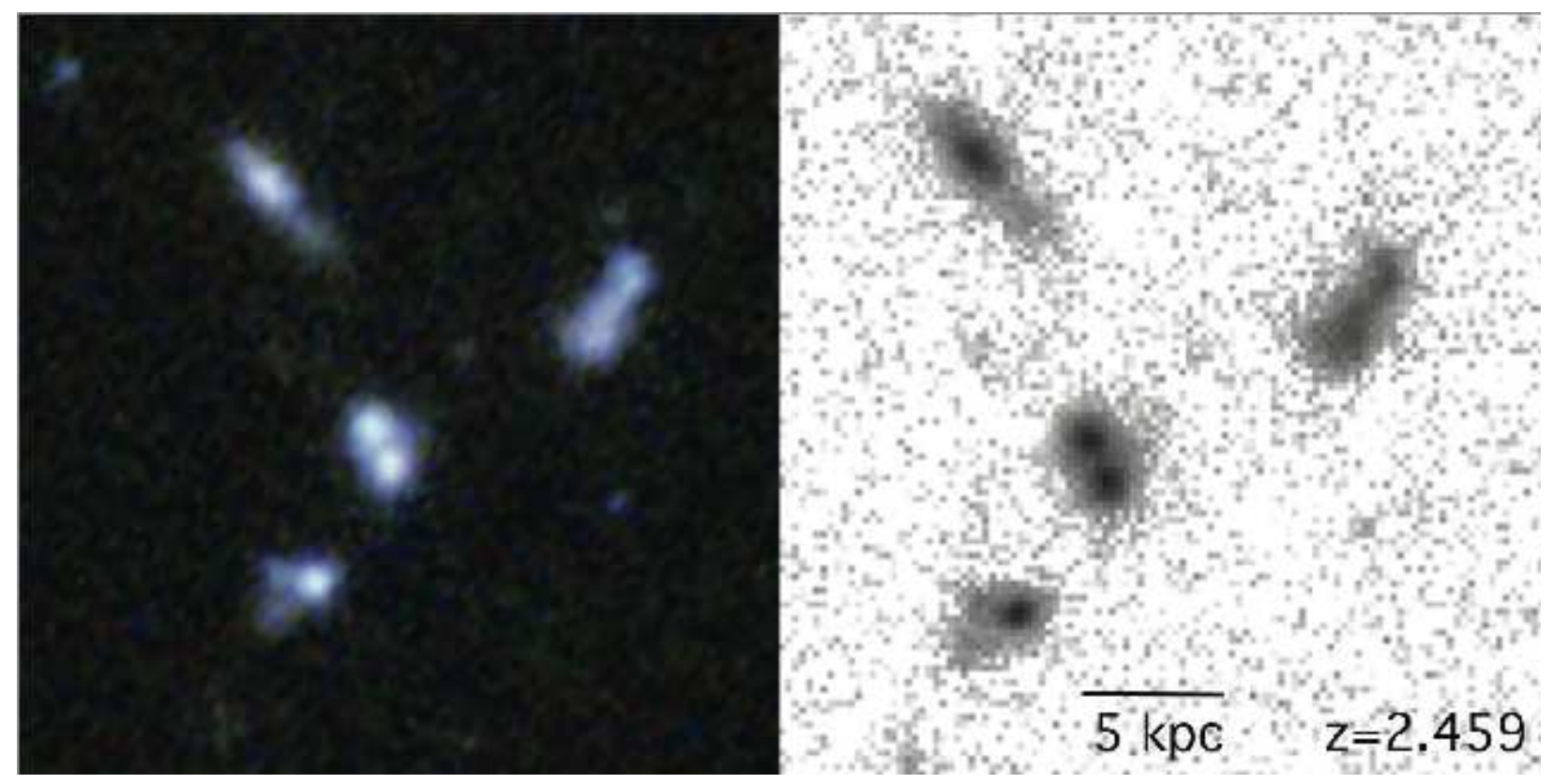

Fig. 15. - Three tadpoles and a double-clump galaxy in the UDF field. The galaxies are: UDF 3583 (upper left), UDF 3527 (upper right), UDF 3508 (bottom), and UDF 3513 (middle). The first three are tadpoles and the fourth is a double-clump. The redshifts in Elmegreen et al. (2007) are 1.87, 1.73, 1.63, and 2.24, respectively. The Rafelski et al. (2009) catalog has two of these galaxies, UDF 3583 with $z=2.08$ and UDF 3527 with $z=2.26$. [Image quality degraded for arXiv] 


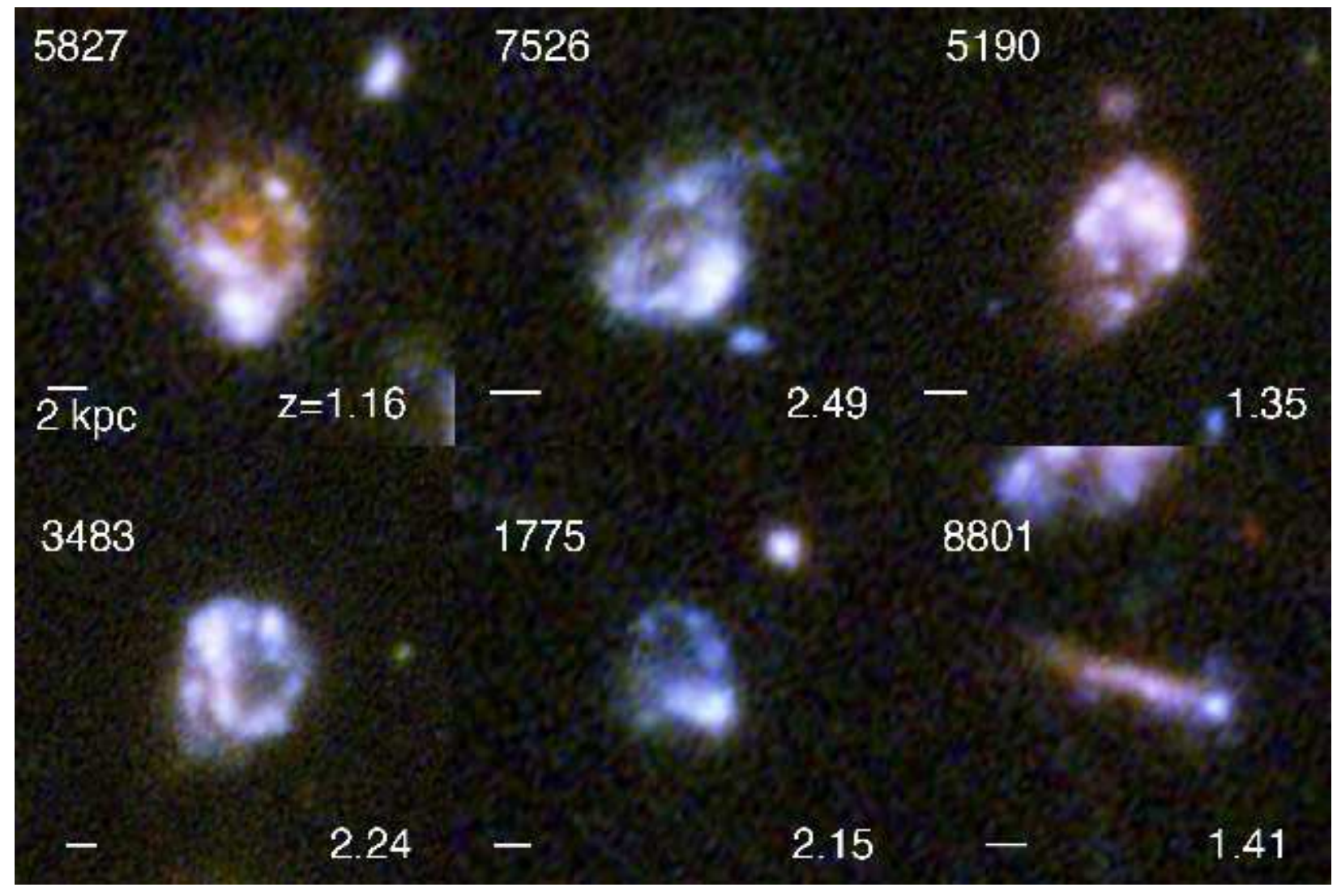

Fig. 16.- Five lop-sided galaxies in the UDF with ring-like structure, plus one straight galaxy, probably an edge-on disk, with a large star-forming clump at one end. The first five galaxies might be classified as tadpoles if they were viewed in the right orientation. The sixth might look like a tadpole if the resolution were a little poorer. [Image quality degraded for arXiv] 